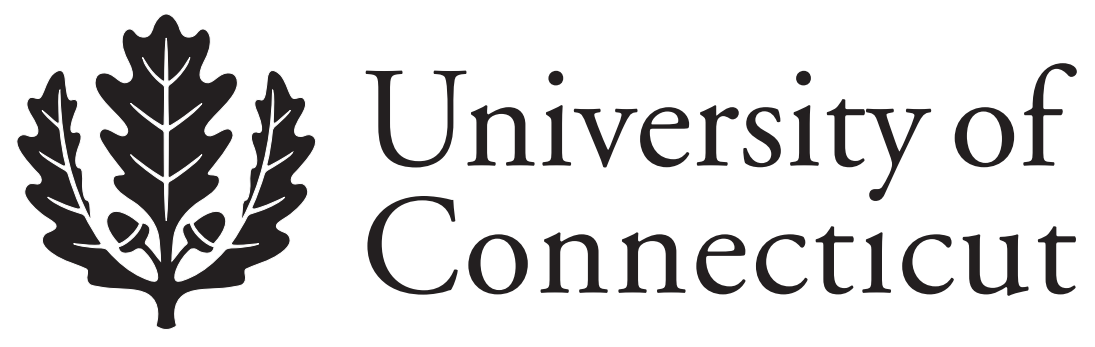

Department of Economics Working Paper Series

Data Envelopment Analysis for Performance Evaluation: A Child's Guide

Subhash C. Ray

University of Connecticut

Lei Chen

University of Connecticut

Working Paper 2009-38

November 2009

341 Mansfield Road, Unit 1063

Storrs, CT 06269-1063

Phone: (860) 486-3022

Fax: (860) 486-4463

http://www.econ.uconn.edu/

This working paper is indexed on RePEc, http://repec.org/ 


\begin{abstract}
In this paper we offer a simple exposition of the neoclassical production theoretic foundations of Data Envelopment Analysis. The concepts of technical efficiency (both input- and output-oriented), scale efficiency, and cost efficiency are explained and the corresponding DEA models are described in details. We offer step-by-step instruction on how to write the codes for solving the various DEA models using the Solver option in the widely accessible MS Excel software. An important feature of this paper is a detailed exposition of how to write various Visual Basic Macro programs for solving DEA problems. We also describe the non-convex Free Disposal Hull (FDH) procedure and the second-stage regression analysis that seeks to account for variation in measured efficiency scores due to external factors.
\end{abstract}

Journal of Economic Literature Classification: C6, D2

Keywords: Efficiency; Linear Programming; Benchmarking; Excel Solver

This paper builds on the material from a keynote address delivered by the first author at the CDE-Swiss Re Workshop on Performance Measurement held by the Delhi School of Economics in Delhi in January 2009. 


\section{DATA ENVELOPMENT ANALYSIS FOR PERFORMANCE EVALUATION: A CHILD'S GUIDE}

In the present age of globalization, efficient utilization of resources is becoming more and more important for firms to survive and prosper in the face of intense competition from both domestic and foreign firms. The usual measure of efficiency often relies on a single indicator like output per worker or business per employee. While easily understood as a convenient measure of performance, it fails to account for the use of other inputs (like materials, energy, and capital) that contribute to the output and constitute the bulk of the production costs of a firm. It is imperative that a comprehensive measure of performance includes all the relevant factors that are important for production.

In evaluating the performance of a business the owners or the managers would typically like to know:

- Is the company making the best use of the resources?

- Is it possible to produce more from the same input bundle? If so, which outputs and how much more?

- Can the firm economize on the resources used? If so, which inputs and by how much?

- Is the firm's input-mix consistent with the relative prices of the inputs? If not, which input should be substituted for what?

- Is the firm of the right size? If not, is it too big or too small?

- Would a potential merger with another specific firm enhance efficiency?

The list goes on.

Even for public sector and not-for-profit private sector agencies engaged in the delivery of public services, efficiency in resource utilization is of critical importance. Provision of public services like education or health care involves use of valuable resources as inputs to produce the desired results as outputs. In that sense, it is like any other production activity. For example, education at the primary level requires teachers, administrative and support staff, and physical resources ranging from class room facilities to textbooks and school supplies. These are the inputs of the educational production process. The outputs on the other hand are the different kinds of cognitive skills acquired by the pupils. It is not unreasonable to treat the school as a firm that uses these inputs to produce the stated outputs. There are, however, several respects in which a public service delivery agency would differ from a commercial firm. First, the outputs here are provided 
to the beneficiaries at zero or minimal cost to them. Second, often there are no market prices available to evaluate the output bundle produced. Even when a subsidized price is charged for any service, it does not reflect either the marginal benefit to the consumer or the marginal cost to the producer. Finally, unlike in the case of a commercial product, there is no obvious criterion like profitability or return on equity that would permit one to evaluate the performance of a public service provider. Such absence of a clear cut measure of performance works against accountability and tends to foster corruption and incompetence. It is important, therefore, to develop suitable measures of performance even when market prices are not available or substantially distorted through subsidization.

In order to evaluate the performance of any decision making unit - be it a commercial firm, a non-profit organization, or a government department - one needs to define a best performance. This can, then, be used as a benchmark for assessment of the actual performance of the unit. Depending upon the context, it could be the maximum output producible from the input actually used by the firm with which one compares the actual output, the minimum cost of producing the observed output of the firm that can be compared to its actual cost, or even simply the maximum output per unit of input actually observed in a sample. Because there are no engineering norms defining how much output can be produced from a given input bundle or the minimum quantities of inputs needed to produce a target output, defining the benchmark becomes an empirical question.

There are two main approaches available for constructing the benchmark technology from observed data. The first is a parametric and econometric approach known as Stochastic Frontier Analysis (SFA) ${ }^{1}$. The other is the nonparametric method of Data Envelopment Analysis (DEA) that uses mathematical programming techniques. The objective of this paper is to familiarize the reader with the economic theoretic foundations of DEA, the various mathematical programming models that are relevant in specific contexts, and how these models can be solved using an easily accessible software, the Excel Solver ${ }^{2}$.

The paper is organized as follows. In section 2 we introduce and distinguish between the three interrelated but different concepts from production economics - effectiveness, productivity, and efficiency. Sections 3 and 4 constitute the core of this paper. Section 3 contains both an intuitive and a technical exposition of the DEA methodology that follows a brief description of the econometric SFA method in section 3.1. Section 4 offers a detailed and step-by-step instruction

\footnotetext{
${ }^{1}$ For a detailed exposition of the SFA methodology see Kumbhakar and Lovell (2000).

2 This paper is not meant to be a substitute for the more rigorous books on DEA like Ray (2004) or Cooper, Seiford, and Tone (2007). Rather, it provides an easier access to the material for those who are more application-oriented and still would like to understand the methodology in general terms.
} 
how to write an appropriate DEA program for measuring output-oriented technical efficiency, scale efficiency, and cost efficiency on the Excel Solver. Guidance is also provided on how to write macros in Visual Basic so that one can evaluate efficiencies of all units at one shot without having to solve one problem at a time. Section 5 explains how one can use the concept of dominance to measure efficiency through Free Disposal Hull (FDH) analysis. Section 6 addresses the question of non-discretionary external factors that can affect the measured efficiency of a firm. Finally, section 7 offers a summing up.

\section{Some Basic Concepts}

In the following paragraphs, we consider a number of alternative measures of performance and describe in details a nonparametric approach that can be applied in a variety of situations and is especially useful when output and input price data are not available.

\subsection{Effectiveness, Productivity, and Efficiency}

A provider of public service is said to be effective when it meets or exceeds a pre set output target. For example, an elementary schooling system is said to be effective when more than $75 \%$ per cent of the pupils tested demonstrate an acceptable level proficiency in some standardized mastery test in reading, writing, and arithmetic. By this measure, the higher the percentage of students "passing" the test, the better performing is the school. A major shortcoming of effectiveness as a measure of performance is that it is based solely on the levels of output and has no relation to the quantities of input used to produce the output observed. A school or a health care facility could be ineffective and fail to reach the goal simply because it has got a very limited budget and cannot afford the resources minimally necessary to achieve the goal. An under-funded agency may be a high performer within the constraints of its limited budget and still remain ineffective.

By far the most commonly used and also the most easily understood measure of performance is productivity. In the simple case of a single output produced from a single input, it is merely the ratio of the output and input quantities. A producer with a higher output per unit of input used is more productive and is deemed to perform in a superior fashion. Consider this simple example involving five firms producing a single output $y$ using a single input, labor $(L)$. The hypothetical input-output quantities are shown in Table 1. 


\begin{tabular}{|l|l|l|l|l|l|}
\hline Firm & A & B & C & D & E \\
\hline Output (y) & 10 & 8 & 16 & 9 & 7 \\
\hline Input (L) & 4 & 7 & 12 & 10 & 9 \\
\hline Labor Productivity & 2.5 & 1.14 & 1.33 & 0.90 & 0.77 \\
\hline
\end{tabular}

Table1. Data for Hypothetical Firms

By this criterion, firm A with the highest labor productivity performs best and firm E does worst. Note that output per worker or labor productivity is itself a descriptive measure summarizing the separate pieces of information about the output and the input quantity of a firm into a single ratio measure. In fact, labor productivity becomes a measure useful for performance evaluation only in a comparative sense. For example, Firm D with labor productivity equal to 0.90 is found to be a relatively poor performer only when compared with firms like A, C, and B.

It is seldom the case, however, that only a single input is used to produce the output. To make this example more realistic we include a second input, capital $(K)$ which was used in conjunction with labor to produce the output levels shown in Table 1 but were not actually reported there. The more complete information on the input bundles and the output levels of the same five firms are now shown in Table 2.

\begin{tabular}{|l|l|l|l|l|l|}
\hline Firm & A & B & C & D & E \\
\hline Output (y) & 10 & 8 & 16 & 9 & 7 \\
\hline Labor Input (L) & 4 & 7 & 12 & 10 & 9 \\
\hline Capital Input (K) & 9 & 3 & 8 & 6 & 8 \\
\hline Labor Productivity & 2.5 & 1.14 & 1.33 & 0.90 & 0.77 \\
\hline Capital Productivity & 1.11 & 2.67 & 2.0 & 1.50 & 0.88 \\
\hline
\end{tabular}

Table2. Input and Productivity Data of Hypothetical Firms

This example clearly illustrates the problem associated with using partial productivity measures to evaluate performance. When productivity is measured as output per unit of capital (rather than by output per worker) firm B emerges as the best performer while firm A slips to the second lowest position. The simple fact of the matter is that the output of a firm incorporates the contribution of both the labor and capital inputs. To use labor productivity to evaluate 
performance amounts to ignoring the contribution of capital and shows the more capital intensive firms in a more favorable light. What we need is an aggregate measure of the inputs and express productivity as the ratio of output to the aggregate input. But how is the aggregate input to be constructed? The task would be simple if input prices were available and all firms faced the same input prices. Suppose that the price of labor was $w=5$ and the rental price of capital was $r=10$. Then a measure of overall productivity would be output per unit of the composite input, i.e., the cost of the input bundle. This of course is the inverse of the average cost. Hence, a firm with a lower average cost is a better performer.

\begin{tabular}{|l|l|l|l|l|l|}
\hline Firm & A & B & C & D & E \\
\hline Output (y) & 10 & 8 & 16 & 9 & 7 \\
\hline Labor Input (L) & 4 & 7 & 12 & 10 & 9 \\
\hline Capital Input (K) & 9 & 3 & 8 & 6 & 8 \\
\hline Cost & 110 & 65 & 140 & 110 & 125 \\
\hline Average Cost & 11 & 8.125 & 8.75 & 12.22 & 17.87 \\
\hline
\end{tabular}

Table3. Cost Data for Hypothetical Firms

In this Table 3, we can use average cost to rank the firms in reverse order of performance. Now firm B with the lowest average cost is the best performer followed closely by firm C. Suppose that output is measured by the number of pupils who complete the primary education. Then a school with the lowest cost per pupil completing elementary school is treated as the best performer and other schools are evaluated using this school as the benchmark.

This approach is quite simple and appeals to common sense. But there are problems. First, when firms face different prices, average cost is not a meaningful criterion because a lower average cost may reflect lower input prices rather than higher productivity. Second, and as is often the case, we may not have appropriate prices of all inputs. In that case, we need to get an aggregate or total factor productivity measure from the output and input quantities alone.

A natural solution would be to take some average of the partial productivities for a measure of total factor productivity. For example, the labor and capital productivities of firm A are

$$
A P_{L}^{A}=\frac{y_{A}}{L_{A}}=\frac{10}{4} \text { and } A P_{K}^{A}=\frac{y_{A}}{K_{A}}=\frac{10}{9} .
$$

Define its total factor productivity as the weighted geometric mean

$$
\operatorname{TFP}^{A}=\left(A P_{L}^{A}\right)^{\beta_{L}}\left(A P_{K}^{A}\right)^{\beta_{K}}
$$


where $\beta_{L}+\beta_{K}=1 ; \beta_{L}, \beta_{K}>0$. Here $\beta_{L}$ and $\beta_{K}$ are, respectively, the weights assigned to labor and capital productivities. For example, if we set $\beta_{L}=0.6$ and $\beta_{K}=0.4$, in this example

$$
\operatorname{TFP}^{A}=(2.5)^{0.6}(1.1)^{0.4}=1.8
$$

For any firm $j(j=A, B, C, D, E)$

$$
\operatorname{TFP}^{j}=\left(\frac{y_{j}}{L j}\right)^{\beta_{L}}\left(\frac{y_{j}}{K_{j}}\right)^{\beta_{K}}=\frac{y_{j}}{X_{j}} ; X_{j}=L_{j}^{\beta_{L}} K_{j}^{\beta_{K}} .
$$

Note that here $X_{j}=L_{j}^{\beta_{L}} K_{j}^{\beta_{K}}$ becomes a measure of aggregate input. We may compare the total factor productivities of two firms B and A through the productivity index

$$
\operatorname{TFPI}_{B, A}=\frac{\operatorname{TFP}_{B}}{T F P_{A}}=\frac{y_{B} / x_{B}}{y_{A} / X_{A}}=\frac{y_{B} / y_{A}}{\left(\frac{L_{B}}{L_{A}}\right)^{\beta_{L}}\left(\frac{K_{B}}{K_{A}}\right)^{\beta_{K}}}=\frac{Q_{y}}{Q_{x}} .
$$

This productivity index is known as the Tornqvist index and is the ratio of an output quantity $\left(Q_{y}\right)$ index and an input quantity index $\left(Q_{X}\right)$. If $T F P I_{B, A}$ exceeds unity, B is more productive than A. Otherwise, $\mathrm{A}$ is more productive.

The weights $\beta_{L}$ and $\beta_{K}$ are of critical importance in the definition of the aggregate input $X$ and can have a significant impact on the how the total factor productivity is measured. When cost information is available, one can use the shares of the labor and capital input in the total cost for these weights. But when cost shares are not known (or they differ across firms), one must use judgment in selecting the weights.

We may now take a closer look at the input aggregator function

$$
X=f(L, K)=L^{\beta_{L}} K^{\beta_{K}} ; \beta_{L}+\beta_{K}=1 ; \beta_{L}, \beta_{K}>0 .
$$

It is easy to see that $f(L, K)$ in $X$ can be regarded as a Cobb Douglas production function exhibiting constant returns to scale. This, however, is a consequence of our decision to take a weighted geometric mean of the partial productivities as a measure of total factor productivity. In fact, we could use any production function exhibiting constant returns to scale and non-negative marginal productivities to define the aggregate input and derive the productivity index.

When the inputs $L$ and $K$ are aggregated through a production function, the total factor productivity of a firm also becomes its technical efficiency index. The production function defines the maximum quantity of output that can be produced from a given input bundle. Thus,

$$
y_{j}^{*}=f\left(L_{j}, K_{j}\right)
$$

is the maximum output that can be produced from the input bundle $\left(L_{j}, K_{j}\right)$. Obviously, the actually observed output from this input bundle must be no greater than the upper limit. That is 


$$
y_{j} \leq y_{j}^{*}=f\left(L_{j}, K_{j}\right) .
$$

We can define the technical efficiency of the firm $j$ as

$$
\tau_{j}=\frac{y_{j}}{y_{j}^{*}} \text {. }
$$

But, when the production function is used to define the aggregate input quantity, it serves also as a measure of total factor productivity. It is important to note, however, that unless the production function is restricted to be homogeneous of degree 1 (i.e., constant returns to scale holds globally), the efficiency measure cannot be treated as a productivity measure. This is because, to serve as a valid input quantity index, apart from being non-decreasing in the individual inputs, $X=f(L, K)$ must also double whenever both inputs are doubled.

We may now consider a more general production function

$$
y^{*}=g(L, K)
$$

where the production function $g(L, K)$ defines the maximum output that can be produced from some specific input bundle $(L, K)$. The technical efficiency of any firm $j$ producing output $y_{j}$ using the input bundle $\left(L_{j}, K_{j}\right)$ is

$$
\tau_{j}=\frac{y_{j}}{y_{j}^{*}}=\frac{y_{j}}{g\left(L_{j}, K_{j}\right)} \leq 1
$$

Note that technical efficiency shows what fraction of the maximum output producible from the input bundle used has been actually produced by the firm $j$. It sets a benchmark that is appropriate for the input quantities actually used.

The following example illustrates why efficiency is a more reasonable measure of performance than effectiveness. Suppose that the production function is

$$
y=2 \sqrt{K L}+\sqrt{L}
$$

and a firm will be considered effective only if $y \geq 20$. Firm 1 produces $y_{1}=28$ units of output and is, therefore, considered effective while firm 2 with $y_{2}=15$ units of output is not. Now suppose that the input bundle of firm 1 is $\left(L_{1}=25, K_{l}=9\right)$. Firm 2's input bundle, on the other hand, is $\left(L_{I}=9, K_{I}=4\right)$. According to the production function specified above, the maximum producible quantities of firms 1 and 2 are, $y_{1}^{*}=35$ and $y_{2}^{*}=15$, respectively. Their corresponding levels of technical efficiency are

$$
\tau_{1}=\frac{28}{35}=0.80 \text { and } \tau_{2}=\frac{15}{15}=1.0 .
$$

It is clear that the maximum producible output from the input bundle used by firm 2 would be lower than the targeted minimum of 20 and no firm could be effective if it had to use this input bundle. On the other hand, firm 1 does exceed the target but is actually under-utilizing its inputs 
producing only $80 \%$ of the maximally producible output quantity. With $100 \%$ technical efficiency, firm 2 is a better performer. It fails to become effective simply because it has got too few resources. In order to make it effective, one must provide it with more resources.

In empirical analysis, measuring technical efficiency to evaluate performance requires estimating the production function $g($.$) that defines the benchmark output level against which the$ actual output is to be compared.

\section{Estimation of the Production Function and Measurement of Efficiency}

As noted before, there are two principal approaches to production function and efficiency measurement that are widely used in the literature. The first is an econometric method known as Stochastic Frontier Analysis (SFA). The other is a non-parametric approach using mathematical programming techniques and is known as Data Envelopment Analysis (DEA).

\subsection{Stochastic Frontier Analysis}

Consider the n-input 1-output production technology characterized by the production function

$$
y^{*}=g\left(x_{1}, x_{2}, \ldots, x_{n}\right) e^{u} .
$$

Here $x=\left(x_{1}, x_{2}, \ldots, x_{n}\right)$ is a bundle of $\mathrm{n}$ inputs and $u$ is a two-sided random error representing favorable or unfavorable random shocks. When $u$ is positive, the maximum output producible from the input bundle $x$ increases. In the opposite case of a negative value of $u$ the maximum producible output is lower. Thus the production frontier itself is random. The actual output $y$ is always on or below the applicable frontier for the realized value of $u$. This can be expressed as

$$
y=g\left(x_{1}, x_{2}, \ldots, x_{n}\right) e^{v-u} ;-\infty \leq v \leq \infty ; u \geq 0 .
$$

Aigner, Lovell, and Schmidt (ALS) (1977) specified a Cobb Douglas form of the function $g($.), the usual Normal distribution $N\left(0, \sigma_{v}^{2}\right)$ for the random shock $v$ and half-Normal distribution $\left|N\left(0, \sigma_{u}^{2}\right)\right|$ for the one-sided technical efficiency term $v$. The log-linear specification of the stochastic production function is

$$
\ln y_{j}=\beta_{0}+\sum_{i=1}^{n} \beta_{i} \ln x_{i j}+v_{j}-u_{j} .
$$

ALS derived the log-likelihood function

$$
\ln L=C-n \ln \sigma+\sum_{j=1}^{n} \ln \Phi\left(-\frac{\varepsilon_{j} \lambda}{\sigma}\right)-\frac{1}{2 \sigma^{2}} \sum_{j=1}^{n} \varepsilon_{j}^{2},
$$

where 


$$
\varepsilon_{j}=v_{j}-u_{j} ; \lambda=\frac{\sigma_{u}}{\sigma_{v}} ; \sigma=\sqrt{\sigma_{u}^{2}+\sigma_{v}^{2}} ; \Phi(.) \text { is the cumulative standard Normal }
$$

distribution function, and $C$ is a constant. The model parameters $\left(\beta=\left(\beta_{0}, \beta_{1}, \ldots, \beta_{n}\right) ; \sigma_{u}^{2}, \sigma_{v}^{2}\right)$ can then be estimated by the maximum likelihood procedure. Jondrow, Materov, Lovell, and Schmidt (1982) have shown that $E\left(u_{j} \mid \varepsilon_{j}\right)=\sigma_{*}\left(\frac{\phi\left(\varepsilon_{j} \lambda / \sigma\right)}{1-\Phi\left(\varepsilon_{j} \lambda / \sigma\right)}-\frac{\varepsilon_{j} \lambda}{\sigma}\right)$ where $\phi($.$) is the density function of$ the standard Normal distribution and $\sigma_{*}=\sqrt{\frac{\sigma_{u}^{2} \cdot \sigma_{v}^{2}}{\sigma^{2}}}$. The estimated technical efficiency of firm $j$ would be

$$
\hat{\tau}_{j}=e^{-\hat{u}_{j}}
$$

where $\hat{u}_{j}=E\left(u_{j} \mid \varepsilon_{j}\right)$.

When a firm produces a vector of outputs rather than a single output, the stochastic frontier production function cannot be used because a production function is not defined in the multiple output case. One has to use a cost function for the analytical framework when multiple outputs are involved. But decomposition of the overall cost efficiency into a technical efficiency and an allocative efficiency component is by no means straight forward.

\subsection{Data Envelopment Analysis and Measurement of Technical Efficiency}

Validity of any estimated stochastic production frontier as the benchmark for evaluating the efficiency of an observed input-output bundle crucially depends on the appropriateness of the functional form of the estimated model. Choice of the preferred functional specification is often arbitrary and is driven by computational simplicity and tractability. Additionally the stochastic distribution of the one-sided inefficiency term (e.g., half-Normal vs. exponential) is a matter of preference for the analyst. The nonparametric method of Data Envelopment Analysis (DEA) introduced by Charnes, Cooper, and Rhodes (CCR) (1978) and further generalized by Banker, Charnes, and Cooper (BCC) (1984) requires no parametric specification of the production frontier and relies on a number of fairly general assumptions about the nature of the underlying production technology. Using a sample of actually observed input-output data and these assumptions, it derives a benchmark output quantity with which the actual output of a firm can be compared for efficiency measurement.

Any production technology transforming an input bundle $x$ into the output bundle $y$ can be characterized by the production possibility set

$$
T=\{(x, y): y \text { can be produced from } x ; x \geq 0 ; y \geq 0\}
$$


In the 1-output case, the frontier or the graph of the technology is defined by the production function

$$
g(x)=\max y:(x, y) \in T .
$$

That is, for any input bundle $x^{0}, g\left(x^{0}\right)$ is the maximum quantity of $y$ that can be produced. An equivalent definition of the production possibility set would be

$$
T=\{(x, y): y \leq g(x) ; x \geq 0 ; y \geq 0\} .
$$

We have seen that in the parametric stochastic frontier analysis one arbitrarily picks up a functional form of $g(x)$. For example, ALS specified the Cobb Douglas form. In DEA, one only makes a number of assumptions about the underlying technology that would be consistent with many different functional forms of the production function but does not select any particular function.

\section{Assumptions about the technology}

1. All actually observed input-output bundles are feasible. That is every input-output combination $\left(x^{j}, y^{j}\right)(j=1,2, \ldots, N)$ in the sample is in $T$.

2. The production possibility set is convex. That is if $\left(x^{1}, y^{1}\right)$ and $\left(x^{2}, y^{2}\right)$ are both feasible, then any weighted average of the two input bundles can produce the corresponding weighted average of the two output bundles. This would be true for any number of feasible input-output bundles. Hence, $\bar{x}=\sum_{j=1}^{N} \lambda_{j} x^{j}$ can produce $\bar{y}=\sum_{j=1}^{N} \lambda_{j} y^{j}$ for any set of non-negative weights $\lambda_{j}(j=1,2, \ldots, N)$ such that $\sum_{j=1}^{N} \lambda_{j}=1$.

3. Inputs are freely disposable. This means that increasing any input without reducing any other input would not cause a decrease in the output. More formally, if $\left(x^{0}, y^{0}\right) \in T$ and $x^{1} \geq x^{0}$, then $\left(x^{1}, y^{0}\right) \in T$. Note that here we are considering a vector inequality in the sense that no element of the $x^{l}$ bundle is smaller than the corresponding element of the $x^{0}$ bundle while some elements may be strictly larger.

4. Outputs are freely disposable. That is if $x^{0}$ can produce $y^{0}$ then it can always produce a smaller output bundle $y^{1} \leq y^{0}$. Formally, if $\left(x^{0}, y^{0}\right) \in T$ and $y^{l} \leq y^{0}$, then $\left(x^{0}, y^{l}\right) \in T$. 


\subsection{Technical Efficiency}

Consider, to start with, a simple technology for producing a single output $(y)$ from a single input $(x)$. In Figure 1 the curve $Y=g(x)$ shows the production function or the graph of the technology. When any point $\left(x^{0}, y^{0}\right)$ lies on this curve, $y^{0}$ is the maximum output that can be produced from input $x^{0}$. Point $P_{A}$ and $P_{B}$ show the actual input-output quantities $\left(x_{A}, y_{A}\right)$ and $\left(x_{B}, y_{B}\right)$, respectively, of two firms $A$ and $B$. Both points lie below the graph. The point $P_{A}^{*}$ on the graph shows that $Y_{A}^{*}$ is the maximum output that can be produced from input $x_{A}$. Thus, $P_{A}^{*}$ shows the benchmark or reference input-output bundle for firm A. Similarly, $P_{B}^{*}$ is the reference bundle for firm B. Define

$$
\varphi_{A}^{*}=\frac{Y_{A}^{*}}{Y_{A}}=\frac{g\left(x_{A}\right)}{Y_{A}} \text {. (17) }
$$

Then a measure of the performance of firm A is its output-oriented technical efficiency

$$
\tau_{y}\left(x_{A}, y_{A}\right)=\frac{1}{\varphi_{A}^{*}} .
$$

The output-oriented technical efficiency shows what proportion of the potential output from $x_{A}$ has actually been realized by firm A. Similarly, the technical efficiency of firm B is measured by the ratio of $Y_{B}$ and $Y_{B}^{*}$. As is apparent from Figure 1, firm B performs better than firm A.

In many situations, however, the output quantity is an assigned task and it is more important to produce the observed output from the smallest quantity of input than to produce the maximum output from the observed input quantity. This leads to an alternative measure of performance the input-oriented technical efficiency. As shown in Figure 2, the minimum input quantity needed to produce output $y_{A}$ is $x_{A}^{*}$. Similarly, $x_{B}^{*}$ is he minimum input quantity needed to produce $y_{B}$. It can be seen that both bundles $\left(x_{A}^{*}, y_{A}\right)$ and $\left(x_{B}^{*}, y_{B}\right)$ lie on the graph of the technology. That is, $g\left(x_{A}^{*}\right)=y_{A}$ and $g\left(x_{B}^{*}\right)=y_{B}$. Define $\theta_{A}^{*}=\frac{x_{A}^{*}}{x_{A}}$ and $\theta_{B}^{*}=\frac{x_{B}^{*}}{x_{B}}$. Then the input-oriented technical efficiencies of the two firms are

$$
\begin{aligned}
& \tau_{x}\left(x_{A}, y_{A}\right)=\theta_{A}^{*} \quad \text { and } \\
& \tau_{x}\left(x_{B}, y_{B}\right)=\theta_{B}^{*} .
\end{aligned}
$$

The input-oriented technical efficiency denotes what proportion of its observed input bundle is actually necessary for producing its observed output. Unless $\tau_{x}$ equals unity, the firm is wasting input. 
In order to numerically compute the technical efficiency of a firm - whether output- or inputoriented - one needs to empirically construct the graph of the technology. In the econometric SFA approach one starts with an explicit specification of a production function and applies the maximum likelihood estimation procedure to estimate the parameters of the model. There are two major advantages of this approach. First, one gets the sampling distribution of the parameter estimates and can use them for hypothesis testing. Second, one can derive the elasticities or other features of the technology from the estimated model analytically. On the downside, validity of the entire SFA analysis is contingent on the correctness of the specified functional form which remains a maintained hypothesis. We now use a simple geometric example to explain how DEA, the nonparametric alternative, can be used to construct the benchmark frontier without any explicit specification of a functional form.

\section{A 1-input 1-output Example}

Consider the data listed in Table 1. In this 1-input 1-output example, the actual input-output bundles of the different firms are shown by the points A through E in Figure 1. By assumption (A1) each of these observed input-output bundles is feasible. Next, by convexity, all points in the closed area ABEDC are also feasible. Finally, by the free disposability assumptions, all points to the right of this area and all points below this enlarged area are also feasible. Hence, the graph of the technology constructed from the data points and the assumptions (A1-A4) is the broken line FACG. The corresponding production possibility set consists of points on or below this graph. Now consider the firm $\mathrm{D}$. The maximum output producible from its input $(\mathrm{x}=9)$ is attained at the point $\mathrm{D}^{*}$ on the graph directly above the point $\mathrm{D}$. The point $\mathrm{D}^{*}$ is a $(0.25,0.75)$ weighted average of the points A and C. Hence the output at $\mathrm{D}$ is $y_{D}^{*}=14.5$. Thus, $\varphi_{D}^{*}=\frac{14.5}{9}$ and $\tau_{\mathrm{y}}(\mathrm{D})=0.6209$.

\section{A 2-input 1-output Example}

The simple geometric approach to construct the graph and to evaluate the output- or inputoriented technical efficiency of a firm described above can be applied only in the case of 1-input 1-output production. Even when only 2-inputs are used to produce a single output, this simple diagrammatic approach fails and one must resort to algebra. To illustrate the algebraic approach we consider the simple 1-output 2-input data shown in Table 2. Suppose that we want to evaluate the performance of firm $E$. Now, by assumption 1, each of the five input-output bundles observed in the sample is feasible. Now construct a weighted average of these input bundles applying 
weights 0.25 to $\left(L_{A}, K_{A}\right), 0.25$ to $\left(L_{C}, K_{C}\right), 0.50$ to $\left(L_{D}, K_{D}\right)$, and zero weight to the other two bundles. The resulting weighted average bundle would be $(\bar{L}, \bar{K})=(9,7.25)$. The corresponding weighted average of the output quantities of these firms would be $\bar{y}=8.75$. Hence, by assumption 2, it is possible to produce 8.75 units of the output from 9 units of labor and 7.25 units of capital. But firm $E$ is using 9 units of labor and 8 units of capital. That is compared to the average input bundle $(\bar{L}, \bar{K})$ it is actually using more capital but no less labor. Hence, by free disposability of inputs (i.e., assumption 3 ) it is possible to produce 8.75 units of the output from the input bundle actually used by firm $E$. It should be emphasized that nowhere have we suggested that 8.75 is the maximum amount of output producible from the actual input bundle of firm $E$. But because 8.75 is a feasible quantity, the maximum cannot be any lower than this feasible quantity. That is, $g\left(L_{E}, K_{E}\right) \geq 8.75$. Hence,

$$
\tau_{E}=\frac{y_{E}}{g\left(L_{E}, K_{E}\right)} \leq \frac{7}{8.75}=0.80
$$

But is there any other weighted average of the observed input bundles that does not require more of either labor or capital than what firm $E$ is using but the corresponding weighted average of the outputs is even higher than 8.75 ?

The answer in this case is in the affirmative. In fact, a different weighting scheme with 0.33 assigned to $\mathrm{A}, 0.67$ to $\mathrm{B}$, and 0.6 to $\mathrm{C}$ would result in an input bundle $\left(L^{*}, K^{*}\right)=(9,8)$ which, coincidentally, is exactly the same bundle that $E$ is using, but the resulting weighted average of the outputs would be $y^{*}=16.46667$. This implies that $g\left(L_{E}, K_{E}\right) \geq 16.46667$. With this inputoutput bundle as the benchmark the technical efficiency of firm $E$ would be measured as

$$
\tau_{E} \leq \frac{7}{13.46667}=0.519802
$$

This, however, raises more questions. First, how did we get these new set of weights? Second, how do we know that there is no other weighting scheme that could result in an even higher value of the weighted average of the outputs without violating the constraint that the weighted average of the inputs should not exceed either the labor or the capital input used by firm $E$ ? Answers to both of these questions lie in the following linear programming problem: 
$\max \varphi$

$\lambda_{A}\left[\begin{array}{l}4 \\ 9\end{array}\right]+\lambda_{B}\left[\begin{array}{l}7 \\ 3\end{array}\right]+\lambda_{C}\left[\begin{array}{c}12 \\ 8\end{array}\right]+\lambda_{D}\left[\begin{array}{c}10 \\ 6\end{array}\right]+\lambda_{E}\left[\begin{array}{l}9 \\ 8\end{array}\right] \leq\left[\begin{array}{l}9 \\ 8\end{array}\right]$

s.t.

$$
\begin{aligned}
& \lambda_{A}(10)+\lambda_{B}(8)+\lambda_{C}(16)+\lambda_{D}(9)+\lambda_{E}(7) \geq \varphi(7) ; \\
& \lambda_{A}+\lambda_{B}+\lambda_{C}+\lambda_{D}+\lambda_{E}=1 ; \\
& \lambda_{A}, \lambda_{B}, \lambda_{C}, \lambda_{D}, \lambda_{E} \geq 0 ; \varphi \text { unrestricted. }
\end{aligned}
$$

In this problem, $\lambda_{A}$ through $\lambda_{E}$ are the weights to be assigned to the individual input-output bundles. The last two constraints ensure that they are all non-negative and that they do add up to $100 \%$. The first two constraints ensure that the weights chosen are such that the weighted average of the observed input bundles does not exceed the input bundle of the firm under evaluation (which is firm $E$ in this example). Finally, the left hand side of the $3^{\text {rd }}$ constraint is the corresponding weighted average of the observed outputs. Because our objective is to maximize $\varphi$, that can be achieved by selecting the $\lambda$ s that maximize the weighted average of the outputs without violating the other constraints. In this particular case the optimal solution of this LP problem yields $\left(\lambda_{A}^{*}=0.33 ; \lambda_{B}^{*}=0.67 ; \lambda_{C}^{*}=0.6 ; \lambda_{D}^{*}=\lambda_{E}^{*}=0 ; \varphi^{*}=1.92\right)$.

Hence, an estimate of $g\left(L_{E}, K_{E}\right)$ is $\varphi^{*} y_{E}=13.47$. We know that there does not exist any other set of weights that would yield a higher value of the output without violating the constraints. If there had been any, the optimal solution would have picked that set of weights. Finally, even though $\varphi$ is unrestricted in sign, $\left(\lambda_{A}=\lambda_{B}=\lambda_{C}=\lambda_{D}=0 ; \lambda_{E}=1 ; \varphi=1\right)$ would be a feasible solution. Hence, $\varphi$ would never be lower than 1 , even though it is unrestricted.

A similar LP problem solved for firm $D$ had an optimal solution with $\varphi^{*}=1.42$, $\lambda_{B}=0.4, \lambda_{C}=0.6$ and the other $\lambda$ s equal to zero. Hence, the estimated technical efficiency of firm $D$ was

$$
\tau_{D}=\frac{1}{1.42}=0.70
$$

For each of the remaining firms $(A, B$, and $C), \varphi^{*}$ was 1 implying that there is no weighted average of the observed input bundles that could yield a weighted average of the outputs exceeding the actual outputs of these firms. Thus, based only on these simple assumptions (1)-(4) and the data, we cannot regard these firms as inefficient. 


\section{A Multiple Output Multiple Input Case}

One of the main appeals of DEA is that unlike the frontier production function analysis, it can easily handle multiple output technologies. It is useful to illustrate this with an example. For this, we modify the input-output data shown in Table 2 by introducing a second output. The revised data are presented in Table 4 below:

\begin{tabular}{|l|l|l|l|l|l|}
\hline Firm & A & B & C & D & E \\
\hline Output 1 $\left(\mathrm{y}_{1}\right)$ & 10 & 8 & 16 & 9 & 7 \\
\hline Output 2 $\left(\mathrm{y}_{2}\right)$ & 6 & 4 & 4 & 8 & 6 \\
\hline Labor Input (L) & 4 & 7 & 12 & 10 & 9 \\
\hline Capital Input (K) & 9 & 3 & 8 & 6 & 8 \\
\hline
\end{tabular}

\section{Table 4. 2-output 2-input Data for 5 Hypothetical Firms}

In order to evaluate the efficiency of firm $E$ in this 2-output example, we solve the revised LP problem

s.t.

$$
\begin{aligned}
& \max \varphi \\
& \lambda_{A}\left[\begin{array}{l}
4 \\
9
\end{array}\right]+\lambda_{B}\left[\begin{array}{l}
7 \\
3
\end{array}\right]+\lambda_{C}\left[\begin{array}{c}
12 \\
8
\end{array}\right]+\lambda_{D}\left[\begin{array}{c}
10 \\
6
\end{array}\right]+\lambda_{E}\left[\begin{array}{l}
9 \\
8
\end{array}\right] \leq\left[\begin{array}{l}
9 \\
8
\end{array}\right] \\
& \lambda_{A}\left[\begin{array}{c}
10 \\
6
\end{array}\right]+\lambda_{B}\left[\begin{array}{l}
8 \\
4
\end{array}\right]+\lambda_{C}\left[\begin{array}{c}
16 \\
4
\end{array}\right]+\lambda_{D}\left[\begin{array}{l}
9 \\
8
\end{array}\right]+\lambda_{E}\left[\begin{array}{l}
7 \\
6
\end{array}\right] \geq \varphi\left[\begin{array}{l}
7 \\
6
\end{array}\right] \\
& \lambda_{A}+\lambda_{B}+\lambda_{C}+\lambda_{D}+\lambda_{E}=1 \\
& \lambda_{A}, \lambda_{B}, \lambda_{C}, \lambda_{D}, \lambda_{E} \geq 0 ; \varphi \text { unrestricted. }
\end{aligned}
$$

In this case, instead of a scalar output, each firm is producing a bundle of two outputs $\left(\mathrm{y}_{1}, \mathrm{y}_{2}\right)$. To accommodate this, we now have two restrictions for the outputs. In the left hand sides of the $3^{\text {rd }}$ and the $4^{\text {th }}$ restrictions, we are constructing weighted averages of these individual output vectors 
of the firms in the sample. The optimal solution of this problem yields $\lambda_{A}^{*}=0.17 ; \lambda_{D}^{*}=0.83$; $\lambda_{B}^{*}=\lambda_{C}^{*}=\lambda_{E}^{*}=0$. The resulting output and input bundles are, respectively, $\left(\overline{y_{1}}=9.17 ; \overline{y_{2}}=7.66\right)$ and $(\bar{L}=9 ; \bar{K}=6.5)$. Note that the weighted average input bundle consists of the same quantity of $L$ and 1.5 fewer units of $K$ compared to the input bundle of firm $E$. On the output side, $\bar{y}_{1}=1.31 y_{1}^{E}$ and $\bar{y}_{2}=1.28 y_{2}^{E}$. The optimal value of $\varphi$ is

$$
\phi^{*}=\min \left\{\frac{\bar{y}_{1}}{y_{1}^{E}} ; \frac{\bar{y}_{2}}{y_{2}^{E}}\right\}=1.28 \text {. }
$$

Note that in the weighted average output bundle $y_{1}$ is $30.95 \%$ higher and $y_{2}$ is $27.78 \%$ higher than the quantities of these outputs produced by firm E. Taking the lower of the two, we find that every output of firm E can be increased by a factor of 1.28 or larger. This is the value of $\varphi^{*}$. In this case, the technical efficiency of firm $E$ is

$$
\tau_{E}=\frac{1}{\varphi^{*}}=0.78 \text {. }
$$

It is easy to see that this is an overly favorable estimate of the efficiency of firm $E$. This is because apart from increasing both outputs by $27.78 \%$ an additional $3.17 \%$ increase would be feasible for output 1 while at the same time use of the capital input could be cut down from 8 to 6.5 units. The technical efficiency measure shown above does not reflect such further output increase or input reduction potential.

\subsection{An Algebraic Formulation of the DEA Optimization Problem}

Let $x^{j}=\left(x_{1}^{j}, x_{2}^{j}, \ldots, x_{n}^{j}\right)$ be the bundle of $\mathrm{n}$ inputs used and $y^{j}=\left(y_{1}^{j}, y_{2}^{j}, \ldots, y_{m}^{j}\right)$ the bundle of m outputs produced by firm $\mathrm{j}(\mathrm{j}=1,2, \ldots, N)$. Suppose that $k$ is one of the observed firms and we wish to measure the technical efficiency of firm $k$. The observed input output bundle of firm $k$ is $\left(x^{k}, y^{k}\right)$. The relevant DEA LP problem would be $\max \varphi$

s.t. $\quad \sum_{j=1}^{N} \lambda_{j} x_{i j} \leq x_{i k}(i=1,2, \ldots, n)$

$$
\begin{aligned}
& \sum_{j=1}^{N} \lambda_{j} y_{r j} \geq \varphi y_{r k}(r=1,2, \ldots, m) ; \\
& \sum_{j=1}^{N} \lambda_{j}=1 ; \lambda_{j} \geq 0(j=1,2, \ldots, N) ; \varphi \text { unrestricted. }
\end{aligned}
$$

The technical efficiency of firm $k$ would be measured by 


$$
\tau_{k}=\frac{1}{\varphi^{*}}
$$

where $\varphi^{*}$ is the optimal solution of the DEA LP problem above.

\section{Output and Input-Oriented Measures of Technical Efficiency}

At this point, it would be useful to formally define the production possibility set constructed from the sample data set $D=\left\{\left(x^{j}, y^{j}\right) ; j=1,2, \ldots, N\right\}$. The sample estimate of the underlying production possibility set $T$ is

$$
S=\left\{(x, y): x \geq \sum_{j=1}^{N} \lambda_{j} x^{j} ; y \leq \sum_{j=1}^{N} \lambda_{j} y^{j} ; \sum_{j=1}^{N} \lambda_{j}=1 ; \lambda_{j} \geq 0(j=1,2, \ldots, N)\right\} .
$$

The set $S$ is also described as the free disposal convex hull of the observed input-output vectors. There are two alternative ways to look at the technical efficiency of a firm that uses the input bundle $x^{0}$ and produces the output bundle $y^{0}$. In what is known as the output-oriented approach, one examines to what extent (if at all) would it be produce to increase the output without requiring to use any additional input. When multiple outputs are involved (i.e., $x^{0}$ is a vector of outputs) without any prior knowledge about the relative significance of the individual outputs in the bundle, one tries to find out what is the maximum equi-proportionate increase possible in all outputs in the bundle. It is understood that in specific cases, some individual outputs could be increased even more than what is implied by the common expansion rate. This was illustrated in the preceding example analyzing the efficiency of firm $E$.

A measure of the output-oriented technical efficiency of a firm with observed input-output bundle $\left(x^{k}, y^{k}\right)$ is

$$
\tau_{k}^{y}=\frac{1}{\varphi^{*},} \text { where } \varphi^{*}=\max \varphi:\left(x^{k}, \varphi y^{k}\right) \in S .
$$

Typically, as in the case of public education or provision of health care, providing the services to more individuals from the actual resources spent is considered more important than lowering the resources spent (keeping the output at its observed level). In such cases, the output-oriented technical efficiency is the appropriate measure of performance. It is an unfortunate fact, however, that government agencies, much like individuals, have limited resources to satisfy competing needs. More resources devoted to education leaves fewer resources for other services like disaster relief or construction of infrastructures. In such cases, if it is agreed that the observed output level meets in some sense an adequate goal, the primary objective would be to reduce the input used to the extent possible without lowering the output. The inputs saved can then be used to meet other 
goals. When input conservation is regarded as more important than expanding outputs, the appropriate measure of performance of firm $k$ would be its input-oriented technical efficiency

$$
\tau_{k}^{x}=\theta^{*}=\min \theta:\left(\theta x^{k}, y^{k}\right) \in S \text {. (26) }
$$

The relevant DEA LP problem for measuring the input-oriented technical efficiency of firm $k$ is

$$
\begin{array}{ll} 
& \min \theta \\
\text { s.t. } \quad & \sum_{j=1}^{N} \lambda_{j} x_{i j} \leq \theta x_{i k}(i=1,2, \ldots, n) \\
& \sum_{j=1}^{N} \lambda_{j} y_{r j} \geq y_{r k}(r=1,2, \ldots, m) ; \\
& \sum_{j=1}^{N} \lambda_{j}=1 ; \lambda_{j} \geq 0(j=1,2, \ldots, N) ; \theta \quad \text { unrestricted. }
\end{array}
$$

Again, it is easy to see that if $\left(x^{k}, y^{k}\right)$ is an actual observation in the sample, $\theta^{*}$ will always be less than or equal to 1 . Using the 2-input, 2-output example from Table 4, the input-oriented DEA LP for firm $E$ had an optimal solution $\lambda_{\mathrm{A}}=0.35948, \lambda_{B}=\lambda_{D}=0.32026, \lambda_{C}=\lambda_{E}=0 ; \theta^{*}=0.76471$. This weighted average of the input bundles of firms $A, B$, and $D$ would reduce both inputs of firm $E$ to only $76.471 \%$ of their existing levels. More over, it would result in no change in output 1 but an increase in the quantity of output 2 by 2.03922 units.

\subsection{Returns to Scale}

None of the four assumptions that we made about the technology had anything to do with returns to scale. Returns to scale is a property of the frontier of the production possibility set. When a small equi-proportionate increase in all inputs causes a more than proportionate increase in all outputs along the frontier, locally increasing returns to scale prevails. Similarly, locally diminishing returns to scale holds when the proportionate increase in outputs is lower than the proportionate increase in inputs. In the case of constant returns to scale, outputs and inputs increase (or decrease) by the same proportion along the frontier. It is possible that the technology exhibits increasing, constant, or deminishing returns to scale along different segments of the frontier. This Variable Returns to Scale (VRS) is the more general assumption about the 
production technology. If, however, one assumes that Constant Returns to Scale (CRS) holds everywhere along the frontier, definition of the production possibility set and the resulting measure of technical efficiency will change.

An implication of the (global) CRS assumption is that if any input-output bundle $(x, y)$ is feasible, so would be the bundle $(t x, t y)$ for any non-negative $t$. Take another look at the definition of the production possibility set $\mathrm{S}$ in (21) above. We know that by convexity, any input-output bundle $(\bar{x}, \bar{y})$ such that $\bar{x}=\sum_{j=1}^{N} \lambda_{j} x^{j}$ and $\bar{y}=\sum_{j=1}^{N} \lambda_{j} y^{j}$ for a set of non-negative $\lambda_{\mathrm{j}} \mathrm{s}$ satisfying $\sum_{j=1}^{N} \lambda_{j}=1$ will be feasible. If additionally we assume that CRS holds, $(t \bar{x}, t \bar{y})$ will also be feasible for every non-negative $t$. Define $\mu_{j}=t \lambda_{j}$. Then, under CRS, $\left(\sum_{j=1}^{N} \mu_{j} x^{j}, \sum_{j=1}^{N} \mu_{j} y^{j}\right)$ is feasible for any set of non-negative $\mu_{j}$ s. In particular, the $\mu_{j}$ s are not restricted to add up to unity.

Hence, under the assumption of CRS, the corresponding construction of the production possibility set would be

$$
S^{C}=\left\{(x, y): x \geq \sum_{j=1}^{N} \lambda_{j} x^{j} ; y \leq \sum_{j=1}^{N} \lambda_{j} y^{j} ; \lambda_{j} \geq 0(j=1,2, \ldots, N)\right\} .
$$

Here the superscript $C$ indicates that CRS has been assumed. Note the absence of the constraint that the $\lambda$ s would have to add up to unity. This equality constraint will also be removed from the output- or input-oriented DEA LP problems when CRS is assumed. Removal of a constraint makes the CRS DEA problems less restrictive than the corresponding VRS models. As a result $\varphi^{*}$ will either be higher or stay the same when compared with the optimal solution of the VRS problem. Similarly, $\theta^{*}$ from the CRS problem will be either strictly lower or equal to what is obtained under VRS. This means that measured technical efficiency under CRS will be less than or equal to what is obtained under the VRS assumption. Moreover, when CRS is assumed, the input- and output-oriented measures will be identical. This is not the case under the VRS assumption.

\section{Scale Efficiency}


In the foregoing discussion of technical efficiency, either the input level or the output level was treated as given and the objective was to either maximize output from the given input or minimize input for producing the given output. Obviously, when the output increases but the input remained unchanged, average productivity increases. Similarly, a reduction in input with unchanged output also raises productivity. Consider output-oriented technical efficiency in the 1 -input 1-output case. If $\tau_{y}\left(x_{0}, y_{0}\right)$ is unity, the ratio

$$
\mathrm{AP}_{0}=\frac{y_{0}}{x_{0}}
$$

is the maximum average productivity that can be attained at the observed input scale $x_{0}$. But suppose that the firm could choose its input level. Then the question would be:

What is the input level $x^{*}$ that maximizes average productivity?

Note that a necessary condition for maximization is

$$
\frac{d A P(x)}{d x}=0
$$

at $x=x^{*}$. But this implies that locally constant returns to scale holds at $x^{*}$. Frisch (1965) described this as the technically optimal production scale. The scale efficiency of a firm using the input $x_{0}$ is measured by comparing the efficient average productivity at $x_{0}$ with the maximum average productivity (attained at $x^{*}$ ). The efficient average productivity at $x_{0}$ is

$$
A P^{*}\left(x_{0}\right)=\frac{g\left(x_{0}\right)}{x_{0}} .
$$

The maximum average productivity is

$$
A P^{*}\left(x^{*}\right)=\frac{g\left(x^{*}\right)}{x^{*}} \text {. }
$$

Thus, scale efficiency at $x_{0}$ is

$$
S E\left(x_{0}\right)=\frac{A P^{*}\left(x_{0}\right)}{A P^{*}\left(x^{*}\right)} \text {. }
$$

The concept of scale efficiency and also its measurement can be easily understood from the diagram in Figure 3. In this 1-input 1-output example, the production function is

$$
g(x)=2 x^{\frac{1}{2}}-4 \text { for } x \geq 4 ; g(x)=0 \text { from } x<4 .
$$

The firm under consideration uses input $x_{0}=9$ to produce output $y_{0}=1$. Its actual average productivity is $A P_{0}=\frac{1}{9}$. But $g\left(x_{0}\right)=2$. Hence, the firm is not on the frontier. The efficient average 
productivity at the observed input level is $A P^{*}\left(x_{0}\right)=\frac{2}{9}$. This is shown by the slope of the line $O Q_{0}$. The maximum average productivity is attained at the point $Q^{*}$. At the optimal production scale $x^{*}=16, g\left(x^{*}\right)=4$ and $A P^{*}\left(x^{*}\right)=\frac{1}{4}$. Hence,

$$
\operatorname{SE}\left(x_{0}\right)=\frac{8}{9} .
$$

Note that Scale efficiency lies between 0 and 1, by construction. In this example, the measured value $\frac{8}{9}$ implies that even at full technical efficiency at its observed input scale of $x_{0}=9$, the average productivity of the firm be(about) $90 \%$ of the maximum average productivity that could be achieved at the optimal input scale $x^{*}=16$.

Now consider the ray through the origin

$$
r(x)=\frac{1}{4} x
$$

which is tangent to the production function $g(x)=2 x^{\frac{1}{2}}-4$ at the point $Q^{*}$. Clearly, at $Q^{*}$ the average and marginal productivities are equal and CRS holds locally. However, if this tangent line itself had been the production function, there would be CRS globally. Ray (2004) called this the pseudo production function. Only one point on this tangent line is feasible and that is the tangency point, $Q^{*}$. Now look at the point $R_{0}$ on this tangent line. Average productivity at $Q^{*}$ is the same as the average productivity at $R_{0}$. Hence,

$$
S E\left(x_{0}\right)=\frac{A P^{*}\left(x_{0}\right)}{A P^{*}\left(x^{*}\right)}=\frac{\frac{Q_{0} x_{0}}{O x_{0}}}{\frac{Q^{*} x^{*}}{O x^{*}}}=\frac{\frac{Q_{0} x_{0}}{O x_{0}}}{\frac{R_{0} x_{0}}{O x_{0}}}=\frac{Q_{0} x_{0}}{R_{0} x_{0}}=\frac{g\left(x_{0}\right)}{r\left(x_{0}\right)} \text {. }
$$

Now, we have already seen that the output-oriented technical efficiency of the firm under evaluation is

$$
\tau_{y}\left(x_{0}, y_{0}\right)=\frac{y_{0}}{g\left(x_{0}\right)}
$$

If, on the other hand, one assumed that the technology exhibited CRS everywhere, the frontier would have been $y=r(x)$ rather than $y=g(x)$. The technical efficiency under the CRS assumption would be

$$
\tau_{y}^{C}\left(x_{0}, y_{0}\right)=\frac{y_{0}}{r\left(x_{0}\right)}
$$


Hence,

$$
S E\left(x_{0}\right)=\frac{g\left(x_{0}\right)}{r\left(x_{0}\right)}=\frac{\tau_{y}^{C}\left(x_{0}, y_{0}\right)}{\tau_{y}\left(x_{0}, y_{0}\right)} .
$$

In the present case, $\tau_{y}^{C}\left(x_{0}, y_{0}\right)=\frac{1}{2 \frac{1}{4}}$ and $\tau_{y}\left(x_{0}, y_{0}\right)=\frac{1}{2}$. Hence, $S E\left(x_{0}, y_{0}\right)=\frac{8}{9}$ as obtained before.

When the output-oriented DEA LP model for firm $\mathrm{E}$ in the 2-input 2-output example shown before was solved under the CRS assumption, the optimal solution was

$$
\lambda_{A}=0.3939 ; \lambda_{B}=\lambda_{C}=\lambda_{E}=0 ; \lambda_{D}=0.7424 ; \varphi^{*}=1.3838 \text {. }
$$

Note that the technical efficiency is strictly lower under the CRS assumption. Further, the $\lambda$ weights do not add up to unity in this case. The CRS technical efficiency of firm E was $\tau_{y}^{C}(E)=0.72$. As seen before, the corresponding VRS efficiency was 0.78 . Hence, the scale efficiency was 0.93 .

\subsection{Measurement and Decomposition of Cost Efficiency}

A widely prevalent misconception among productivity analysts is that DEA may be used in public sector and non-profit applications where prices are either unavailable or irrelevant. For market entities trying to minimize cost or maximize profit, one should use an econometrically estimated parametric cost or profit function to construct the benchmark. The reality is that the choice between DEA and SFA is decided by one's willingness (or otherwise) to rely on a specific functional form of the technology and not by the availability of market prices. In this section we show how one can use DEA to evaluate the minimum cost of producing the observed output (bundle) for a firm at given market prices of inputs. Comparing the minimum cost with the actual cost incurred by the firm yields a measure of its cost efficiency.

A firm minimizes cost when it uses an input bundle that can produce the target output at the lowest cost at the applicable input prices. The choice, therefore, is only among those input bundles that can actually produce the desired output. The set of input bundles $(x)$ that can produce a specified output $\left(y^{0}\right)$ is the input requirement set

$$
V\left(y^{0}\right)=\left\{x:\left(x, y^{0}\right) \in T\right\} \text {. }
$$


In the standard textbook example, the input requirement set consists of all input bundles that lie on or above the isoquant for the given output, $y^{0}$. A 1-output 2-input example of the input requirement set for the output level 10 is

$$
V(10)=\left\{\left(x_{1}, x_{2}\right): f\left(x_{1}, x_{2}\right) \geq 10\right\} .
$$

For example, the input requirement set for the production function

$$
f\left(x_{1}, x_{2}\right)=\sqrt{x_{1}}+2 \sqrt{x_{2}}
$$

is

$$
V(10)=\left\{\left(x_{1}, x_{2}\right): \sqrt{x_{1}}+2 \sqrt{x_{2}} \geq 10\right\}
$$

Suppose that the vector of input prices faced by the firm is $w^{0}=\left(w_{10}, w_{20}\right)$. Then the minimum cost is

$$
C\left(w^{0}, y^{0}\right)=\min w^{0} x: \sqrt{x_{1}}+2 \sqrt{x_{2}} \geq 10 .
$$

If the actual input bundle used by the firm is $x^{0}=\left(x_{10}, x_{20}\right)$ then the actual cost is

$$
C^{0}=w_{10} x_{10}+w_{20} x_{20}
$$

The cost efficiency of the firm can be measured as $\gamma=\frac{C\left(w^{0}, y^{0}\right)}{C^{0}}$.

Figure 4 provides a simple graphical illustration of measuring cost efficiency. In the diagram the curve $f\left(x_{1}, x_{2}\right)=y^{0}$ is the isoquant for the output level $y^{0}$. All points on and above this curve are in $V\left(y^{0}\right)$. Point A shows the actual input bundle of the firm. The expenditure line EF through the point $\mathrm{A}$ is the iso-cost line

$$
w_{10} x_{1}+w_{20} x_{2}=C^{0}
$$

If the firm could eliminate its input-oriented technical inefficiency by scaling down both inputs by the factor $\tau_{\mathrm{x}}(=\theta)$ it could move to the input bundle $\mathrm{B}$ on the isoquant. The iso-cost line GH through B shows the cost of this technically efficient bundle $\left(x_{1}^{T}=\theta x_{1}^{0} ; x_{2}^{T}=\theta x_{2}^{0}\right)$ :

$$
w_{10} x_{1}^{T}+w_{20} x_{2}^{T}=C^{T}
$$

Once technical inefficiency has been removed, the only way any further reduction in cost (if possible) must through a trade off between the inputs. Given the input prices, the cost efficient 
point $C$ is the point of tangency between the isoquant and the iso-cost line $\mathrm{JK}$. The minimum cost is

$$
C^{*}=C\left(w^{0}, y^{0}\right)=w_{10} x_{1}^{*}+w_{20} x_{2}^{*}
$$

In this diagram, cost efficiency is

$$
\gamma=\frac{C\left(w^{0}, y^{0}\right)}{C^{0}}=\frac{O D}{O A} .
$$

This can be decomposed as

$$
\gamma=\frac{O D}{O A}=\frac{O B}{O A} \cdot \frac{O D}{O B} .
$$

Here $\frac{O B}{O A}=\theta$ is the technical efficiency and $\frac{O B}{O A}=\alpha$ is the allocative efficiency.

In order to operationalize this measurement and decomposition of cost efficiency, one needs to construct the relevant isoquant for the target output level. When an explicit specification of the production function is available, one can simply look at the graph of the implication function

$$
f\left(x_{1}, x_{2}\right)=y^{0} .
$$

In DEA, however, we proceed through the input requirement set for $y^{0}$ :

$$
V\left(y^{0}\right)=\left\{x:\left(x, y^{0}\right) \in T\right\} .
$$

If we use the empirically constructed set, $S$, for $T$ :

$$
V\left(y^{0}\right)=\left\{x: x \geq \sum_{j=1}^{N} \lambda_{j} x^{j} ; y^{0} \leq \sum_{j=1}^{N} \lambda_{j} y^{j} ; \sum_{j=1}^{N} \lambda_{j}=1 ; \lambda_{j} \geq 0(j=1,2, \ldots, N)\right\} .
$$

The minimum cost, $C\left(w^{0}, y^{0}\right)$ can be obtained as

$$
C^{*}=\min \sum_{i=1}^{n} w_{i 0} x_{i j}
$$

s.t. $\quad \sum_{j=1}^{N} \lambda_{j} x_{i j} \leq x_{i k}(i=1,2, \ldots, n)$

$$
\begin{gathered}
\sum_{j=1}^{N} \lambda_{j} y_{r j} \geq y_{r 0}(r=1,2, \ldots, m) ; \\
\sum_{j=1}^{N} \lambda_{j}=1 ; \lambda_{j} \geq 0(j=1,2, \ldots, N) .
\end{gathered}
$$




\section{A 1-output 2-input Example}

Consider the data from Table 3 above. Suppose that we wish to evaluate the cost efficiency of firm D in the sample. It uses the input bundle $\left(L_{D}=10, K_{D}=6\right)$ and produces output $y_{D}=9$. At input prices $(w=5, r=10)$ its actual cost is 110 . To obtain its minimum cost we solove the DEA LP problem:

$$
\min C=5 L+10 \mathrm{~K}
$$

s.t. $4 \lambda_{A}+7 \lambda_{B}+12 \lambda_{C}+10 \lambda_{D}+9 \lambda_{E} \leq L$;

$$
\begin{aligned}
& 9 \lambda_{A}+3 \lambda_{B}+8 \lambda_{C}+6 \lambda_{D}+8 \lambda_{E} \leq K ; \\
& 10 \lambda_{A}+8 \lambda_{B}+16 \lambda_{C}+9 \lambda_{D}+7 \lambda_{E} \geq 9 ; \\
& \lambda_{A}+\lambda_{B}+\lambda_{C}+\lambda_{D}+\lambda_{E}=1 ; \\
& \quad \lambda_{A}, \lambda_{B}, \lambda_{C}, \lambda_{D}, \lambda_{E} \geq 0 .
\end{aligned}
$$

The optimal solution for this LP problem is

$$
\left\{\left(\lambda_{A}^{*}=\lambda_{D}^{*}=\lambda_{E}^{*}=0 ; \lambda_{B}^{*}=0.875, \lambda_{C}^{*}=0.125\right) ;\left(L^{*}=7.635, K^{*}=3.635\right) ; C^{*}=74.375 .\right\} .
$$

Thus, the cost efficiency of firm $D$ is

$$
\gamma_{D}=\frac{74.375}{110}=0.676
$$

This implies that it is possible to produce the observed output level of $D$ at only $67.6 \%$ of its actual cost. The input-oriented technical efficiency obtained by solving the relevant DEA LP

problem was $\theta_{D}=0.707$. Hence, the allocative efficiency was $\alpha_{D}=\frac{\gamma_{D}}{\theta_{D}}=0.956$. This implies that about $30 \%$ of the cost could be reduced by simply eliminating technical inefficiency without altering the input ratio. A further $5 \%$ reduction could be achieved by changing the input-mix.

\section{DEA on Excel Solver}

In this section we offer a step by step instruction on how to write the programs for various kinds of DEA models for Excel Solver. Specifically, first we show examples of DEA models for measuring output-oriented VRS technical efficiency of individual firms in the 1-output 2-input and 2-output 2-input cases. Then we explain how one can write Macro commands in Visual Basic 
in order to measure efficiency of all units in the sample all at once. Then we describe the programs for measuring cost efficiency both for a single firm and for all firms at once using a Macro.

1-output 2-input ourput oriented Technical Efficiency

We consider the empirical example of one-output two-input output-oriented DEA TE model with the data from Table 2. The model is specified in equation (20). To solve this LP problem in Excel, we first import the data to an Excel spreadsheet as shown in the Solver Figure 1. Column A records the firm's name. Column B shows the firm's number according to its appearance in column A. Column $\mathrm{C}$ to column $\mathrm{E}$ shows the original data for the output and inputs. In column F, we define the lambdas. Therefore, cells F4 to F8 are the changing variables lambda 1 through lambda 5. In columns $\mathrm{H}$ to $\mathrm{J}$, on row 4, we define the left hand side of the constraints. Specifically, the command we need to type in cell $\mathrm{H} 4$ is

“=SUMPRODUCT(C4:C8,\$F\$4:\$F\$8)”.

Next, we can copy and paste this command to cells I4 to J4 on the same row. The symbol "\$" in the above command works as a cell address fixing indicator. It will fix the column letter or row number to the right of it when the command is being copied and pasted to another cell. The unfixed column letter and/or row number will be automatically updated according to where the original command is copied and pasted to.

In column $\mathrm{H}$, on row 6 , we identify the firm for which we want to evaluate the technical efficiency. In Excel, the VLookup function searches for value in the left-most column of table_array and returns the value in the same row from another column in the table_array based on the index_number. The syntax for the VLookup function is ${ }^{3}$ :

VLookup(value, table_array, index_number).

On row 7, we find the associated serial number for the firm using the command

\footnotetext{
${ }^{3}$ An excellent reference for advanced Excel functions is Powell and Baker (2007)
} 
“=VLOOKUP(H6,A4:B8,2)".

In the present case, as shown in Solver Figure 1 below, the array (B4:B8) contains the names of the firms and their serial numbers. In cell H6 we have entered the firm name, E. Hence, the VLOOKUP command finds the row in the first column of the specified array that contains the entry E. This is Row 7. Then it finds the entry in the $7^{\text {th }}$ row of the $2^{\text {nd }}$ column of the array. This is the value 5 in cell B7.

On row 8, we define the sum of all lambdas, so the command in cell H8 is "=SUM(F4:F8)". On row 9 in the same column, we define value of phi which is the objective function of the LP problem. Note that cell H9 is also a changing variable. Cell H10 shows the technical efficiency of the selected firm. It equals the inverse of the maximized phi in cell H9. Thus the formula in cell H10 is " $=1 / \mathrm{H} 9 "$.

On row 5, we need to fill in the right hand side of the constraints. In Excel, the Index function returns the value of an element in a table or an array selected by the row and column number indexes. The syntax for the Index function is:

$$
\text { Index(array, row_number, column_number), }
$$

where array is a range of cells or table; row_number is the row number in the array to use to return the value; column_number is the column number in the array to use to return the value (optional if the array is a single column array).

Because the right hand side of the output constraint is defined as the actual output of the selected firm multiplied by phi, in cell H5 we type command "=INDEX(C4:C8,H7)*H9”.

In the present case, the array is a single column (C4:C8). The entry in cell $\mathrm{H} 7$ identifies the relevant row (Row 5). Thus, it picks up the output of firm E, the unit under evaluation. This is multiplied by the entry in cell H9 which contains the value of 'phi'.

The right hand side of the input constraint is just the actual input for the selected firm. So in cell I5 we type "=INDEX(D4:D8,\$H\$)", and then copy and paste this command to cell J5 on the same row.

We have finished with inputting information to the spreadsheet, and now we are going to fill in the solver parameters. For this, we first select "Solver" from the menu "Data", a Solver 
Parameters window will pop-up. In the 'Set Target Cell' box in this window, we type "H9"; among the 'Equal To' options, we select "Max"; in the 'By Changing Cells' box, we type "F4:F8, H9". Then we click the icon "Add" to impose constraints. In the "Add Constraints' window, we type "H4" in the 'Cell Reference' box; in the box next to it, we select " $>=$ "; in the 'Constraint' box, we type "H5". Once we have done that, we click "Add" to save current constraint and continue to the next constraint. Now we type "I4:J4" in the Cell Reference box on the left; select " $<=$ " in the middle box; type "I5:J5" in the Constraint box on the right. Click "Add", and start typing the third constraint, which is to type "H8" in the Cell Reference box; select "=" in the middle box; type "1" in the Constraint box. Now click "OK" to go back to the Solver Parameters window, and then click "Options". In the Solver Options windows, check "Assume Linear Model" and "Assume Non-negative", and then click "OK". The last step, click "Solve" on the right upper corner, Excel Solver will start solving the problem and report the result to us once it's done. If Solver finds an optimal solution, the optimized value of phi will be shown in cell H9, and the technical efficiency for the selected firm will be shown in cell H10.

In this example, we first evaluate firm E's technical efficiency by typing "E" in cell H6 and solving the LP problem in Solver. The result is shown in the following figure:

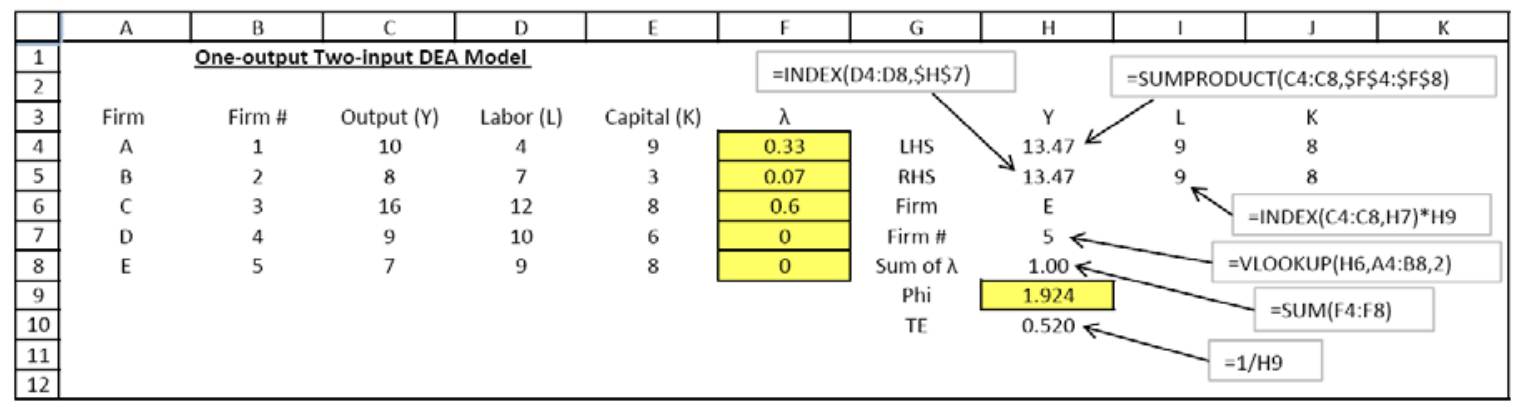

Solver Figure 1: Measuring TE of firm E

We can evaluate firm D's technical efficiency by simply changing "E" to "D" in cell H6 and resolving the LP problem in Solver (without changing the Solver Parameter setup). The result is shown in the following figure:

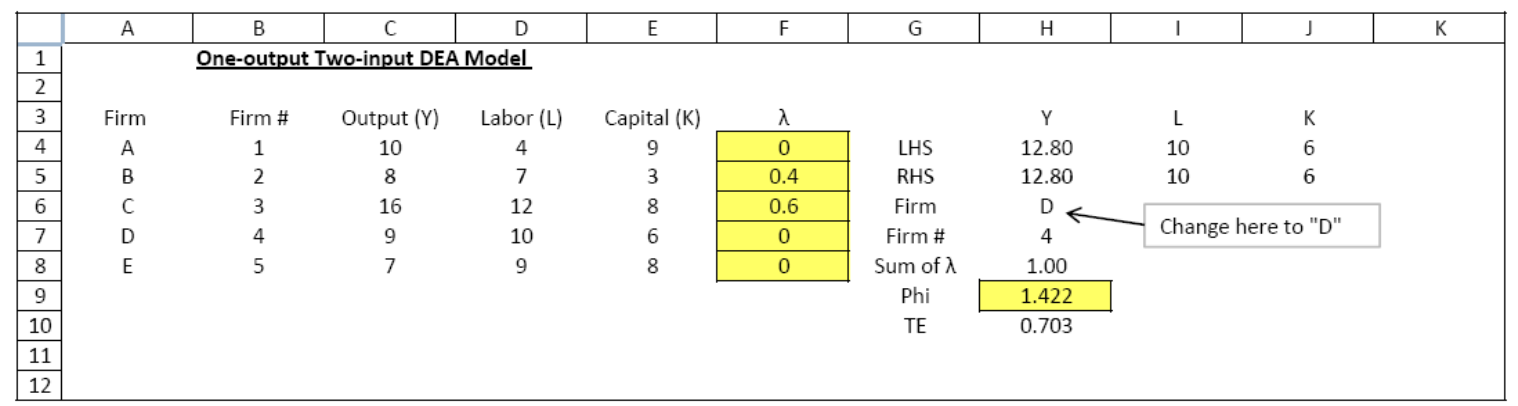




\section{Solver Figure 2. Measuring the TE of Firm D}

\section{2-output 2-input Example}

Next, we consider the empirical example of two-output two-input output-oriented DEA TE model with the data from Table 4. The model is specified in equation (21). To solve this LP problem in Excel, we first import the data to an Excel spreadsheet as shown in the Solver Figure 2. Column A records the firm's name. Column B shows the firm's serial number according to its appearance in column A. Column $\mathrm{C}$ to column $\mathrm{F}$ shows the original data for outputs and inputs. In column G, we define the lambdas. Therefore, cells G4 to G8 are the changing variables lambda 1 through lambda 5 . In columns I to $\mathrm{L}$, on row 4, we define the left hand side of the constraints. Specifically, the command we need to type in cell I4 is

"=SUMPRODUCT $(\mathrm{C} 4: \mathrm{C} 8, \$ \mathrm{G} \$ 4: \$ \mathrm{G} \$ 8) "$, then we can copy and paste this command to cells J4 to L4 on the same row.

In column I, on row 6 , we define the firm for which we want to evaluate the technical efficiency. On row 7, we find the associated serial number to the firm using the command "=VLOOKUP(I6,A4:B8,2)". On row 8, we define the sum of all lambdas, so the command in cell H8 is "=SUM(G4:G8)". On row 9 in the same column, we define value of phi, which is the objective function of the LP problem. Notice that cell H9 is also a changing variable. Cell I10 shows the technical efficiency of the selected firm. It equals the inverse of the maximized phi in cell I9. Thus the formula in cell $\mathrm{H} 10$ is " $=1 / \mathrm{I} 9 "$ ".

On row 5, we need to fill in the right hand side of the constraints. Because the right hand side of each output constraint is defined as the actual output of the selected firm times phi, in cell I5 we type command "=INDEX $(\mathrm{C} 4: \mathrm{C} 8, \$ \mathrm{II} 7)^{*} \$ \mathrm{I} \$ 9 "$, and then copy and paste this command to cell J5. The right hand side of each input constraint is just the actual input for the selected firm. So in cell K5 we type "=INDEX(E4:E8,\$I7)", and then copy and paste this command to cell L5.

We have finished with inputting information to the spreadsheet, and now we are going to fill in the solver parameters. Now we select "Solver" from the menu "Data", a Solver Parameters window will pop-up. In the Set Target Cell box, we type "I9"; in Equal To options, we select "Max"; in By Changing Cells box, we type "G4:G8, I9". Then we click icon "Add" to impose constraints. In the Add Constraints window, we type "I4:J4" in the Cell Reference box; in the box next to it, we select ">="; in the Constraint box, we type "I5:J5". Once we have done that, we click "Add" to save current constraint and continue for the next constraint. Now we type "K4:L4" in the Cell Reference box on the left; select " $<=$ " in the middle box; type "K5:L5" in the Constraint box on the right. Click "Add", and start typing the third constraint, which is to type 
"I8" in the Cell Reference box; select "=" in the middle box; type "1" in the Constraint box. Now click "OK" to go back to the Solver Parameters window, and then click "Options". In the Solver Options windows, check "Assume Linear Model" and "Assume Non-negative", and then click "OK". The last step, click "Solve" on the right upper corner, Excel Solver will start solving the problem and report the result to us once it's done. If Solver finds an optimal solution, the optimized value of phi will be shown in cell I9, and the technical efficiency for the selected firm will be shown in cell I10.

In this 2-output 2-input example, we evaluate firm E's technical efficiency by typing "E" in cell I6 and solving the LP problem in Solver. The result is shown in the following figure:

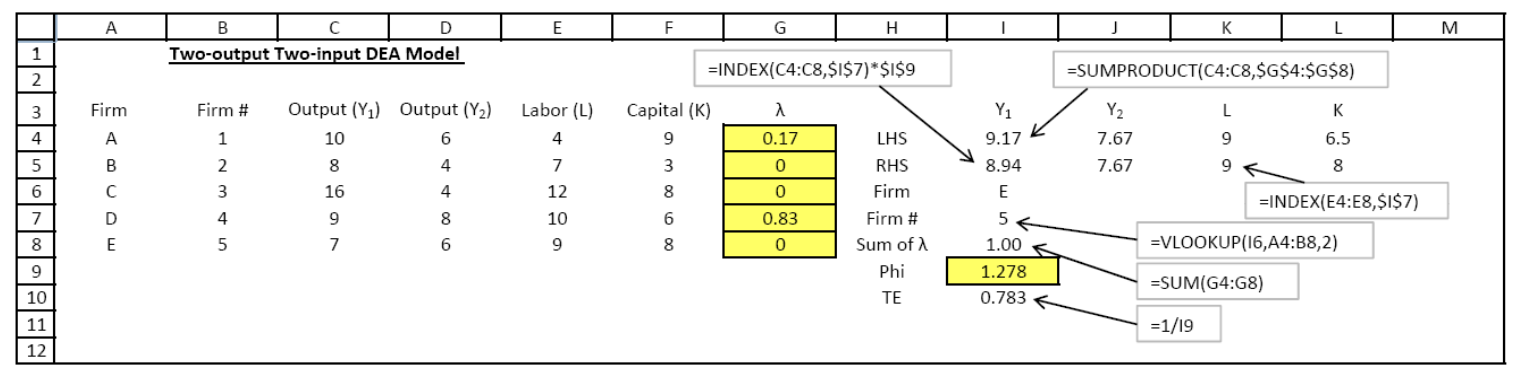

Solver Figure 3: Measuring TE of Firm E (Multiple-output, Multiple-input case)

In the above examples, we show how one can solve the DEA efficiency evaluation problems for the individual firms - one firm at a time. However, when the sample size is large this can be quite time consuming and burdensome. It is useful to write a set of Macro commands that will program Solver to evaluate the efficiencies of all firms in a single run. In the next section we use a sample data set from Indian pharmaceutical firms in an example that explains how to write a Visual Basic Macro for measuring output-oriented technical efficiency. 


\section{$\underline{\text { A Visual Basic Macro }}$}

Empirical Example of Using Excel Solver to Solve DEA Models

We consider the output-oriented DEA TE model and use sample data from a number of Indian pharmaceutical companies in the empirical example. This data set includes 80

observations. We consider one output $(Y)$ and four inputs (material $M$, fuel $F$, labor $L$, and capital $K)$. The DEA model can be expressed as the following:

$$
\begin{array}{ll} 
& \max \varphi \\
& \sum_{j=1}^{N} \lambda_{j} Y_{j} \geq \varphi Y_{0} ; \\
& \sum_{j=1}^{N} \lambda_{j} M_{j} \leq M_{0} ; \sum_{j=1}^{N} \lambda_{j} F_{j} \leq F_{0} ; \\
& \sum_{j=1}^{N} \lambda_{j} L_{j} \leq L_{0} ; \sum_{j=1}^{N} \lambda_{j} K_{j} \leq K_{0} ; \\
& \sum_{j=1}^{N} \lambda_{j}=1 ; \lambda_{j} \geq 0 ;(j=1,2, \ldots, N) .
\end{array}
$$

To solve the above LP problem in Excel, we first import the data to an Excel spreadsheet as shown in the Solver Figure 3. Column A records the firm number. Column B to column F shows the original data for the output and inputs. In column $\mathrm{G}$, we define the lambdas.

Therefore, cells G4 to G83 are the changing variables lambda 1 through lambda 80 . In columns I to $\mathrm{M}$, on row 4, we define the left hand side of the constraints. Specifically, the command we need to type in cell I4 is "=SUMPRODUCT(B4:B83,\$G\$4:\$G\$3)", then we can copy and paste this command to cells J4 to M4 on the same row. The symbol "\$" in the above command works as a cell address fixing indicator. It will fix the column letter or row number to the right of it when the command is being copied and pasted to another cell. The unfixed column letter and/or row number will be automatically updated according to where the original command is copied and pasted to.

In column I, on row 7, we define the firm number for which we want to evaluate the technical efficiency. On row 9, we define the sum of all lambdas, so the command in cell I9 is "=SUM(G4:G83)". On row 11 in the same column, we define value of phi, which is the objective function of the LP problem. Notice that cell I11 is also a changing variable. Cell I13 shows the technical efficiency of the selected firm. It equals the inverse of the maximized phi in cell I11. Thus the formula in cell I13 is " $=1 / \mathrm{I} 11 "$ ". 
On row 5, we need to fill in the right hand side of the constraints. Because the right hand side of the output constraint is defined as the actual output of the selected firm times phi, in cell I5 we type command "=INDEX(B4:B83,I7)*I11". The right hand side of the input constraint is just the actual input for the selected firm. So in cell J5 we type "=INDEX(C4:C83,\$I\$)", and then copy and paste this command to cells K5 to M5 on the same row.

We have finished with inputting information to the spreadsheet, and now we are going to fill in the solver parameters. First, we need to make sure Solver is available for use. In Excel 2007, in the main menu tab on the top, if we select "Data", we should see "Solver" in the subgroup of "Analysis" on the rightmost. If it is not there, we have to do the following: (i) go to the left-upper corner of the window, click "Office Button" once, and then click "Excel Options" at the bottom; (ii) in the Excel Options window, click "Add-Ins" in the left menu column, and then in the Manage box, select "Excel Add-ins" and click "Go"; (iii) In the Add-Ins available box, select the "Solver Add-in" check box, and then click "OK".

Now we select "Solver" from the menu "Data", a Solver Parameters window will pop-up. In the Set Target Cell box, we type "I11"; in Equal To options, we select "Max"; in By Changing Cells box, we type "G4:G83, I11". Then we click icon "Add" to impose constraints. In the Add Constraints window, we type "I4" in the Cell Reference box; in the box next to it, we select ">="; in the Constraint box, we type "I5". Once we have done that, we click "Add" to save current constraint and continue for the next constraint. Now we type "J4:M4" in the Cell Reference box on the left; select " $<=$ " in the middle box; type "J5:M5" in the Constraint box on the right. Click "Add", and start typing the third constraint, which is to type "I9" in the Cell Reference box; select "=" in the middle box; type " 1 " in the Constraint box. Now click "OK" to go back to the Solver Parameters window, and then click "Options". In the Solver Options windows, check "Assume Linear Model" and "Assume Non-negative", and then click "OK". The last step, click "Solve" on the right upper corner, Excel Solver will start solving the problem and report the result to us once it's done (which takes less than one second in most of cases). If Solver finds an optimal solution, the optimized value of phi will be shown in cell I11, and the technical efficiency for the selected firm will be shown in cell I13.

Following the above steps, we can investigate the technical efficiency for each individual firm in the sample. However, it becomes tedious when the total number of firms is large. One solution to this problem is that we can run a Visual Basic macro program in Excel to solve the technical efficiencies once for all. To import the VB macro program, we first need to have the Developer tab on the main menu. If it is not there, we can do the following: (i) go to the leftupper corner of the window, click "Office Button" once, and then click "Excel Options" at the 
bottom; (ii) in the Excel Options window click "Popular" in the left menu column then select "Show Developer tab in the Ribbon" check box on the right, and then click "OK".

Now we have the tab Developer showing on the menu. We click "Developer", and then "Insert" in the "Controls" sub-group. Under the "ActiveX Controls", we click the leftmost icon on the first row, which is also called "Command Button". The arrow cursor now becomes a fine cross cursor. Move the cursor to any blank place on the spreadsheet we are working with, and then click and drag to create an icon for the VB macro program on the spreadsheet. By default, the caption of the icon is "CommandButton1". We can change it by right click it and then select "Properties" from the menu and change the caption to, for example, "Run Macro". Now double click the command button we created, we go to the VB programming for code editing. Between the lines of "Private Sub" and "End Sub", we type in the following code:

For Unit $=1$ To 80

Range("I7") = Unit

SolverSolve UserFinish:=True

Range("N" \& Unit + 3) = Range("I13")

Next Unit

Next, in the Microsoft Visual Basic window, we select "Tools" from the main menu, and then click "References". In the "References - VBAProject" window, select "SOLVER" check box and then click "OK". If the "SOLVER" check box is not shown in that window, we need to do the following: (i) click "Browse..." in that window; (ii) in the "Add Reference" window, look in "Local Disk (C:)" then "Program Files" then "Microsoft Office" then "Office 12" then "Library" then "SOLVER", and then select "Microsoft Office Excel Files" at the "Files of type" window; (iii) select the file "SOLVER" in the "Add Reference" window and click "Open"; (iv) click "OK" in the "References - VBAProject" window.

Now we go back to the Excel spreadsheet we are working on. Click "Design Mode" on the menu once, and then click the command button (Run Macro) we created. The VB macro program should start running and technical efficiency scores for all firms will show up in column $\mathrm{O}$, on the corresponding rows. To save our work, we need to save the Excel file as an "Excel Macro-Enabled Workbook". 


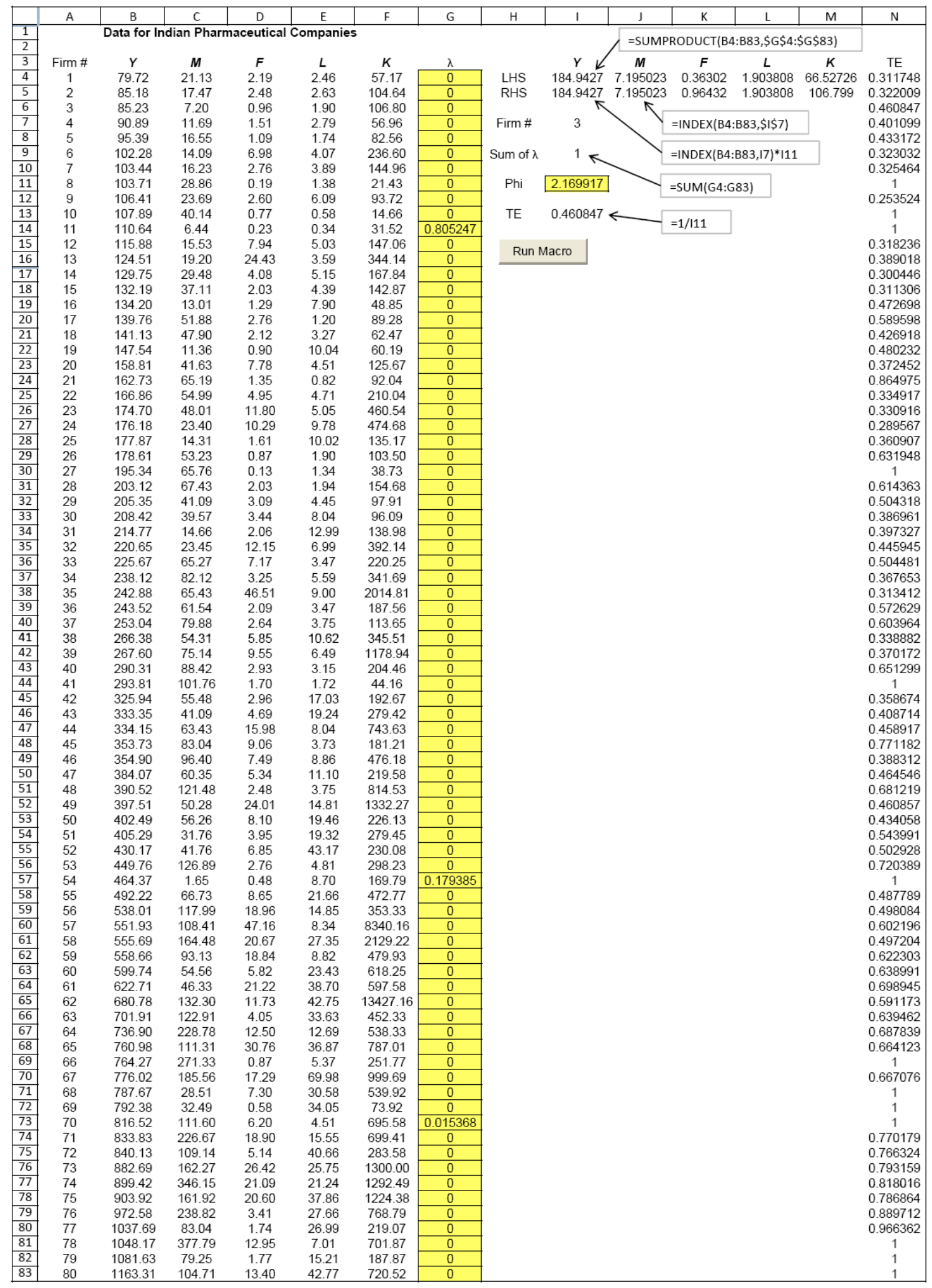

Solver Figure 3. Technical Efficiencies of Indian Pharmaceutical Firms 


\section{Example of Measuring Cost Efficiency}

We consider the empirical example of one-output two-input cost DEA model with the data from Table 3. The model is specified in equation (52). To solve this LP problem in Excel, we first import the data to an Excel spreadsheet as shown in the Solver Figure 4. Column A records the firm's name. Column B shows the firm's number according to its appearance in column A. Column $\mathrm{C}$ to column $\mathrm{E}$ shows the original data for the output and inputs. Column $\mathrm{F}$ records the total cost for each firm given the input prices $w_{\mathrm{L}}=5$ ans $w_{\mathrm{K}}=10$. In column $\mathrm{G}$, we define the lambdas. Therefore, cells G4 to G8 are the changing variables lambda 1 through lambda 5. In columns I to K, on row 4, we define the left hand side of the constraints. Specifically, the command we need to type in cell I4 is "=SUMPRODUCT(C4:C8,\$G\$4:\$G\$8)", then we can copy and paste this command to cells J4 to K4 on the same row.

In column I, on row 7, we define the firm for which we want to evaluate the cost efficiency. On row 8 , we find the associated number for the firm using the command "=VLOOKUP(I7,A4:B8,2)". On row 9, we define the sum of all lambdas, so the command in cell I9 is "=SUM(G4:G8)". On row 10 in the same column, we record the actual total cost. The command in cell I10 is "=INDEX(F4:F8,I8)". Cell I1 1 shows the optimal total cost of the selected firm. Therefore the command in cell $\mathrm{I} 11$ is "=SUMPRODUCT(J5:K5,J6:K6)". We define the DEA cost efficiency as the ratio of the actual cost to the optimal cost. Thus the command in cell $\mathrm{I} 12$ is " $=\mathrm{I} 11 / \mathrm{I} 10 "$.

On row 5, we need to fill in the right hand side of the constraints. Because the right hand side of the output constraint is the actual output of the selected firm, in cell I5 we type command "=INDEX(C4:C8,I8)". The right hand side of the input constraint is the optimal quantity of each input for the selected firm. Notice that cells J5 and K5 are also changing variables. We record the input prices on row 6 under columns $\mathrm{J}$ and $\mathrm{K}$.

We have finished with inputting information to the spreadsheet, and now we are going to fill in the solver parameters. Now we select "Solver" from the menu "Data", a Solver Parameters window will pop-up. In the Set Target Cell box, we type "I11"; in Equal To options, we select "Min"; in By Changing Cells box, we type "G4:G8,J5:K5". Then we click icon "Add" to impose constraints. In the Add Constraints window, we type "I4" in the Cell Reference box; in the box next to it, we select ">="; in the Constraint box, we type "I5". Once we have done that, we click "Add" to save current constraint and continue for the next constraint. Now we type "J4:K4" in the Cell Reference box on the left; select " $<=$ " in the middle box; type "J5:K5" in the Constraint box on the right. Click "Add", and start typing the third constraint, which is to type "I9" in the Cell Reference box; select "=" in the middle box; type "1" in the Constraint box. Now click 
"OK" to go back to the Solver Parameters window, and then click "Options". In the Solver Options windows, check "Assume Linear Model" and "Assume Non-negative", and then click "OK". The last step, click "Solve" on the right upper corner, Excel Solver will start solving the problem and report the result to us once it's done. If Solver finds an optimal solution, the minimized value of total cost will be shown in cell I11, and the cost efficiency for the selected firm will be shown in cell I12.

In this example, we first evaluate firm D's cost efficiency by typing " $D$ " in cell I7 and solving the LP problem in Solver. The result is shown in the following figure:

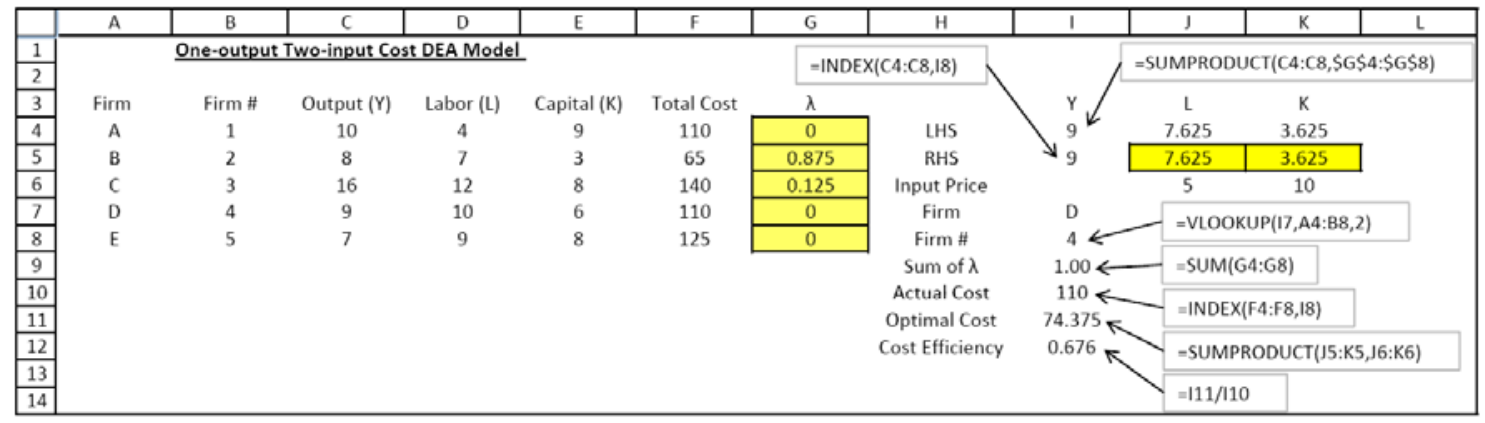

Solver Figure 4. Measuring Cost Efficiency of Firm D 


\section{A VBA Macro for Measuring Cost Efficiency}

In this example, we solve a Cost DEA model and apply the data for Indian manufacturing industry in the empirical example. This data set includes 22 observations. The input-outputs are the average (or per establishment data) obtained from the Annual Survey of Industries for the year 2004. We consider one output $(Y)$ and five inputs (capital $K$, production labor $L_{1}$, nonproduction labor $L_{2}$, fuel $F$, and material $M$ ). We also have the price information for each input ( $w_{k}, w_{1}, w_{2}, w_{f}$, and $w_{m}$ are price indices for the above five inputs respectively). The DEA model can be expressed as the following:

$$
\begin{array}{ll}
\min & w_{k} K+w_{1} L_{1}+w_{2} L_{2}+w_{f} F+w_{m} M \\
& \sum_{j=1}^{N} \lambda_{j} Y_{j} \geq Y_{0} ; \sum_{j=1}^{N} \lambda_{j} K_{j} \leq K ; \\
\text { s.t. } \quad & \sum_{j=1}^{N} \lambda_{j} L_{1 j} \leq L_{1} ; \sum_{j=1}^{N} \lambda_{j} L_{2 j} \leq L_{2} ; \\
& \sum_{j=1}^{N} \lambda_{j} F_{j} \leq F ; \sum_{j=1}^{N} \lambda_{j} M_{j} \leq M ; \\
& \sum_{j=1}^{N} \lambda_{j}=1 ; \lambda_{j} \geq 0 ;(j=1,2, \ldots, N) .
\end{array}
$$

To solve the above LP problem in Excel, we first import the data to an Excel spreadsheet as shown in the Solver Figure 5. Column A records the state number. Column B records the abbreviation of each state. Column $\mathrm{C}$ to column $\mathrm{G}$ shows the original data for the output and inputs. Column I to column $\mathrm{M}$ shows the associated input price indices for the five inputs. In column, we calculate the actual total cost for each state. In column $\mathrm{O}$, we define the lambdas. Therefore, cells $\mathrm{O} 4$ to $\mathrm{O} 25$ are the changing variables lambda 1 through lambda 22. In columns Q to V, on row 4, we define the left hand side of the constraints. Specifically, the command we need to type in cell Q4 is "=SUMPRODUCT(C4:C25,\$O\$4:\$O25)", then we can copy and paste this command to cells R4 to V4 on the same row. The symbol "\$" in the above command works as a cell address fixing indicator. It will fix the column letter or row number to the right of it when the command is being copied and pasted to another cell. The unfixed column letter and/or row number will be automatically updated according to where the original command is copied and pasted to.

In column $\mathrm{Q}$, on row 10, we define the state number for which we want to evaluate the technical efficiency. On row 12 in the same column, we define the sum of all lambdas, so the command in cell Q12 is " $=\mathrm{SUM}(\mathrm{O} 4: \mathrm{O} 25) "$. 
In columns $\mathrm{Q}$ to $\mathrm{V}$, on row 5, we need to fill in the right hand side of the constraints. Because the right hand side of the output constraint is the actual output of the state selected, in cell Q5 we type command "=INDEX(C4:C25,Q10)". The right hand side of the input constraint is just the optimal input for the selected state. So in columns $\mathrm{R}$ to $\mathrm{V}$, on row 6 , we define the optimal level of inputs for the selected state. Please note that we do not type in any expressions in cells R6 toV6 on row 6 . Instead, we define them as changing cells.

In columns $\mathrm{R}$ to $\mathrm{V}$, on row 8 , we define the input price indices for the selected state. Specifically, in cell R8 we type command “=INDEX(I4:I25,\$Q\$10)", then we copy and paste this command to cells $\mathrm{S} 8$ to V8 on the same row.

Once we define the optimal input levels and input price indices for a selected state, we can get the minimum total cost for the state. In column $\mathrm{Q}$, on row 14, we define the minimum total cost by typing command "=SUMPRODUCT(R5:V5,R8:V8)". On row 16 in the same column, we define the actual total cost by typing command "=INDEX(N4:N25,Q10)". At last, we define the cost efficiency of the selected state by typing command "=Q14/Q16" into cell Q18.

We have finished with inputting information to the spreadsheet, and now we are going to fill in the solver parameters. We select "Solver" from the menu "Data", a Solver Parameters window will pop-up. In the Set Target Cell box, we type "O14"; in Equal To options, we select "Min"; in By Changing Cells box, we type "O4:O25, R5:V5". Then we click icon "Add" to impose constraints. In the Add Constraints window, we type "Q4" in the Cell Reference box; in the box next to it, we select ">="; in the Constraint box, we type "Q5". Once we have done that, we click "Add" to save current constraint and continue for the next constraint. Now we type "R4:V4" in the Cell Reference box on the left; select "<=" in the middle box; type "R5:V5" in the Constraint box on the right. Click "Add", and start typing the third constraint, which is to type "O12" in the Cell Reference box; select "=" in the middle box; type " 1 " in the Constraint box. Now click "OK" to go back to the Solver Parameters window, and then click "Options". In the Solver Options windows, check "Assume Linear Model" and "Assume Non-negative", and then click "OK". The last step, click "Solve" on the right upper corner, Excel Solver will start solving the problem and report the result to us once it's done (which takes less than one second in most cases). If Solver finds an optimal solution, the minimized total cost will be shown in cell Q14, and the cost efficiency for the selected state will be shown in cell Q18.

Following the above steps, we can investigate the cost efficiency for each individual state in the sample. We can run a Visual Basic macro program in Excel to solve the cost efficiencies once for all in the following way. From the menu on the top, we click "Developer", and then "Insert" in the "Controls" sub-group. Under the "ActiveX Controls", we click the leftmost icon 
on the first row, which is also called "Command Button". The arrow cursor now becomes a fine cross cursor. Move the cursor to any blank place on the spreadsheet we are working with, and then click and drag to create an icon for the VB macro program on the spreadsheet. By default, the caption of the icon is "CommandButton1". We can change it by right click it and then select "Properties" from the menu and change the caption to, for example, "Run Macro". Now double click the command button we created, we go to the VB programming for code editing. Between the lines of "Private Sub" and "End Sub", we type in the following code:

For Unit $=1$ To 22

Range("Q10") = Unit

SolverSolve UserFinish:=True

Range("W" \& Unit + 3) = Range("Q18")

Next Unit

Next, in the Microsoft Visual Basic window, we select "Tools" from the main menu, and then click "References". In the "References - VBAProject" window, select "SOLVER" check box and then click "OK". If the "SOLVER" check box is not shown in that window, we need to do the following: (i) click "Browse..." in that window; (ii) in the "Add Reference" window, look in "Local Disk (C:)" then "Program Files" then "Microsoft Office" then "Office 12" then "Library" then "SOLVER", and then select "Microsoft Office Excel Files" at the "Files of type" window; (iii) select the file "SOLVER" in the "Add Reference" window and click "Open"; (iv) click "OK" in the "References - VBAProject" window.

Now we go back to the Excel spreadsheet we are working on. Click "Design Mode" on the menu once, and then click the command button (Run Macro) we created. The VB macro program should start running and cost efficiency scores for individual states will show up in column W, on the corresponding rows. To save our work, we need to save the Excel file as an "Excel Macro-Enabled Workbook". 


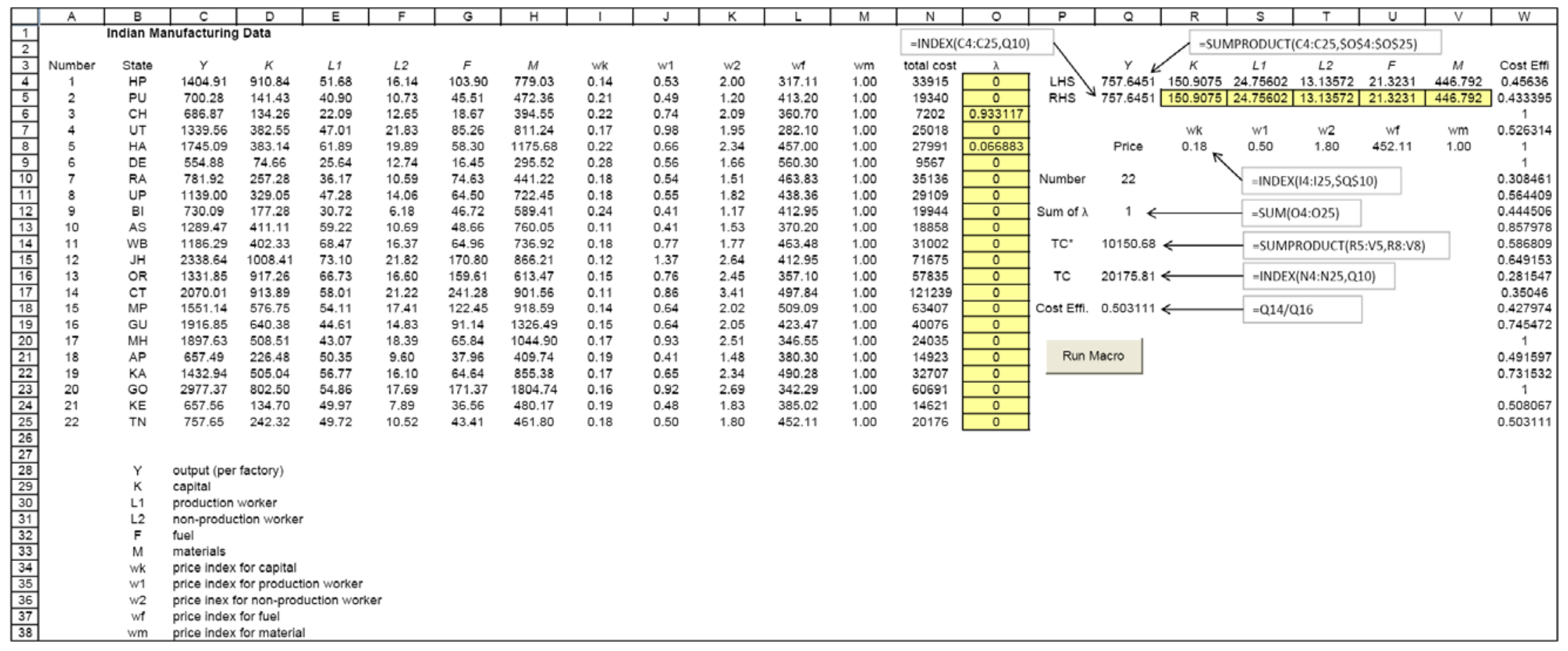

Solver Figure 5. Example of Meausring Cost Efficiency in Manufacturing in the Indian States in the year 2004 


\section{Relaxing the Convexity Assumption: Free Disposal Hull Analysis}

Among the four assumptions made about the technology, convexity is the strongest. The benchmark input-output bundle constructed from a suitably weighted average of the observed input-output data is usually not one of the observed bundles. This may leave the practitioner uneasy because no firm in the sample is actually found to have produced the benchmark output bundle from the weighted average input bundle. By contrast, if a benchmark is an actually

observed bundle the target is evidently feasible and efficiency measured against any such target is clearly more persuasive.

Deprins, Simar, and Tulkens (1984) introduced a revised version of the standard DEA model which became known as Free Disposal Hull (FDH) analysis. Their simple modification was to restrict each $\lambda_{j}$ to be a $0-1$ binary variable. The FDH version of the output-oriented VRS DEA problem (19) is

$\max \varphi$

$$
\begin{array}{ll}
\text { s.t. } \quad & \sum_{j=1}^{N} \lambda_{j} x_{i j} \leq x_{i k}(i=1,2, \ldots, n) \\
& \sum_{j=1}^{N} \lambda_{j} y_{r j} \geq \varphi y_{r k}(r=1,2, \ldots, m) \\
& \sum_{j=1}^{N} \lambda_{j}=1 ; \lambda_{j} \in\{0,1\} ;(j=1,2, \ldots, N) ; \varphi \text { unrestricted. }
\end{array}
$$

Note that because each $\lambda$ is either 0 or 1 and at the same time they add up to 1 , only one $\lambda$ would be 1 and all others will be zero. That means that a single observed input-output bundle is selected as a bench mark for comparison for measuring the efficiency of firm $k$. The restrictions ensure that the benchmark firm will not use any input in greater quantity than firm $k$. Nor would it produce a smaller amount of any output.

The problem in (27) is an integer programming problem. In general, integer programming problems are more complicated than a standard LP problem. But, as shown in Ray (2004, chapter 6) there exists a very simple way to obtain the optimal solution of the FDH problem. 


\section{Taking Account of Non-discretionary Variables}

The quantity of output produced from a given input bundle often depends on a number of factors over which the firm has no control. These can be designated as non-discretionary variables. In agricultural production, the same input bundle even at its best utilization would produce a higher or lower output depending on the amount of rainfall. The farmer has no control over it. In education the same bundle of school inputs would produce a higher output (measured by standardized test scores) when the level of education or socioeconomic status of the parents of the average pupil is higher. Such 'environmental' variables cause the frontier to move up or down. Unlike the two-sided random shock in a stochastic frontier model, however, these variables are observable and can be explicitly accounted for in measuring the technical efficiency of a firm.

There are two different approaches to deal with non-discretionary variables in DEA. In the first, they are directly incorporated in the DEA model itself. For this, one must first classify the non-discretionary variables as favorable or unfavorable. A favorable variable is one that increases the maximum output producible from a given input bundle. An unfavorable variable, by contrast, lowers the maximum producible output from the same input bundle. It is assumed that an increase in the favorable variable or a decrease in the unfavorable variable cannot lower output. The standard DEA models are suitably modified in the light of this assumption. Suppose that in addition to the $\mathrm{n}$ inputs and $\mathrm{m}$ outputs we have the information on the level of one favorable variable $(f)$ and one unfavorable variable $(u)$ for each firm in the sample. Then the revised output-oriented VRS DEA LP model for firm $k$ would become

$\max \varphi$

$$
\begin{array}{ll}
\text { s.t. } \quad & \sum_{j=1}^{N} \lambda_{j} x_{i j} \leq x_{i k}(i=1,2, \ldots, n) \\
& \sum_{j=1}^{N} \lambda_{j} y_{r j} \geq \varphi y_{r k}(r=1,2, \ldots, m) \\
& \sum_{j=1}^{N} \lambda_{j} f_{j} \leq f_{k} \\
& \sum_{j=1}^{N} \lambda_{j} u_{j} \geq u_{k} \\
& \sum_{j=1}^{N} \lambda_{j}=1 ; \lambda_{j} \geq 0(j=1,2, \ldots, N) ; \varphi \text { unrestricted. }
\end{array}
$$


Because firm $k$ has no less of the favorable variable and no more of the unfavorable variable than the hypothetical firm constructed from the weighted average of the observed bundles, it should be able to produce as much output as this weighted average would.

In the other approach, the non-discretionary variables are treated as separable from the inputs and output of the firm. They shift the frontier, which is a function of the controllable inputs only, like Hicks-neutral technical change. One uses the usual inputs and outputs of a firm to obtain a DEA efficiency score. In a second stage regression these efficiency score is treated as the dependent variable and regressed on the non-discretionary variables. An advantage of this approach is that one can estimate how a change in any one discretionary variable affects the measured efficiency of a firm. Moreover, one need not classify them as favorable or unfavorable beforehand. If it is a favorable factor, it should come out with a positive coefficient in the fitted regression. Similarly, an unfavorable factor would have a negative coefficient. There is an econometric problem with this regression, however. The DEA efficiency scores are by construction bounded at unity from above. It is, therefore, a limited dependent variable. A simple least squares regression is in appropriate for such a model and the more careful analyst uses a Tobit regression ${ }^{4}$.

\section{A Summing Up}

In this paper we have offered a detailed introduction to the core DEA models that can be used to measure technical efficiency, scale efficiency, and overall cost efficiency of a firm. The principal appeal of DEA lies in the fact that it creates a benchmark based entirely on observed data for comparison and performance evaluation. In particular, there is no prior assumption about the nature of factor substitutability that is inherent in an explicit specification of the function form of the production or cost function. Of course, the analyst needs to ensure that all of the data come from firms that are technologically homogenous. This, it should be pointed out, is often misinterpreted to be a special problem for DEA alone. In fact, one should not attempt to fit a production function econometrically unless there is reason to believe that the data come from the same data generating process. We have paid special attention to what is effectively a tutorial on how to write the Excel programs (especially those using VBA Macros) for different kinds of DEA models. Once the readers master the art of writing these programs, they can set up and solve

\footnotetext{
${ }^{4}$ While methodologically correct the intuitive meaning of such a regression is not always clear because there is no obvious censoring of the dependent variable that would rationalize a Tobit model.
} 
all kinds of DEA problems (even those that have not been included in this paper) without having to rely on the availability of a prefabricated software.

Finally, over the past three decades since its original formulation in the OR/MS literature, DEA has matured into a full blown nonparametric analytical methodology that provides an alternative to the parametric econometric approach to efficiency measurement. There are various other applications of DEA that measure profit efficiency, productivity growth over time, measuring efficiency in the presence of both good and bad outputs, and so on. It is hoped that this introduction will encourage and enable the more enterprising reader to move on to the more rigorous and complete books like Färe, Grosskopf, and Lovell (1994), Ray (2004), and Cooper, Seiford, and Tone (2007), to name a few. 


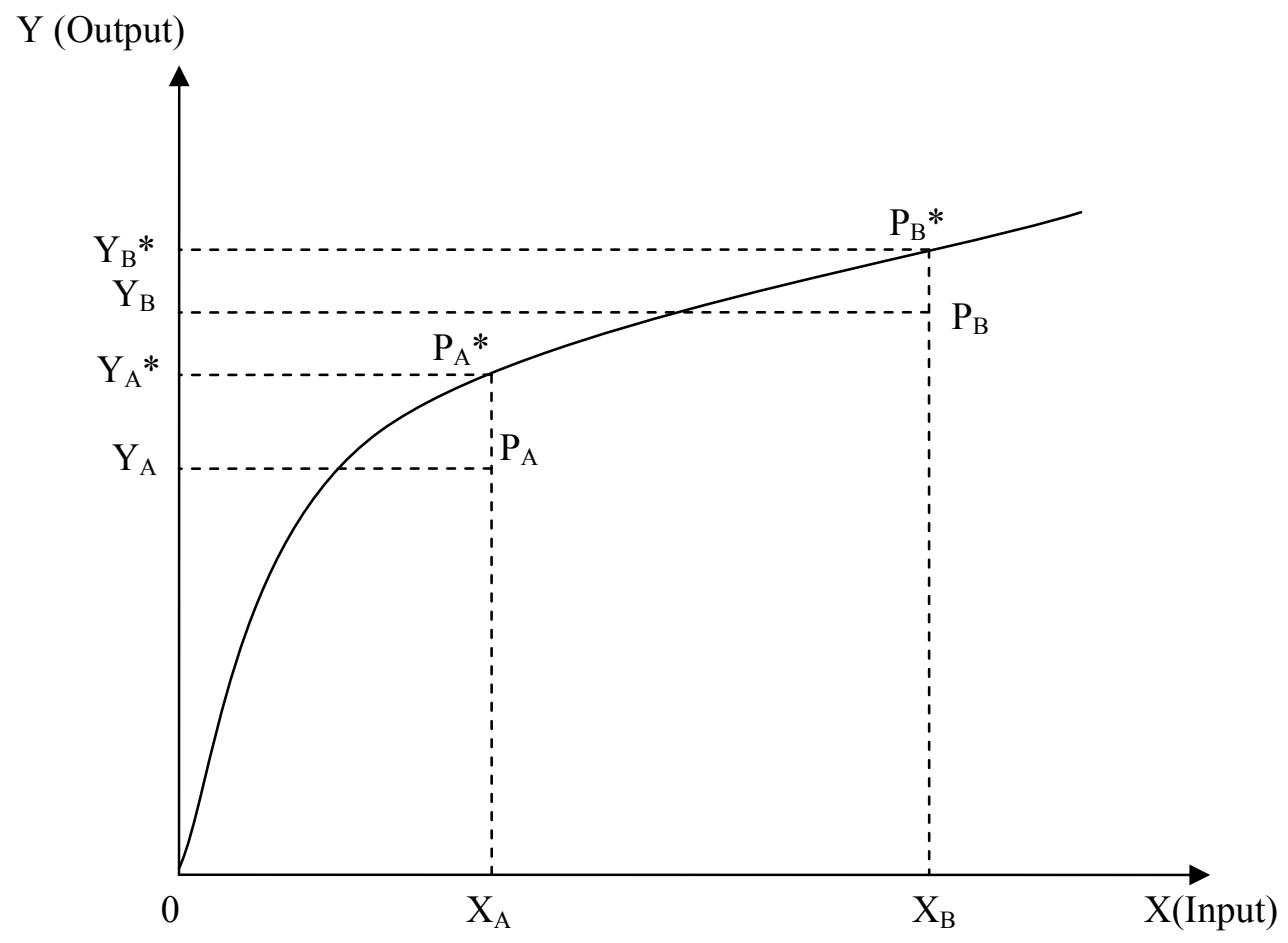

Figure 1 Measuring Output-oriented Technical Efficiency 


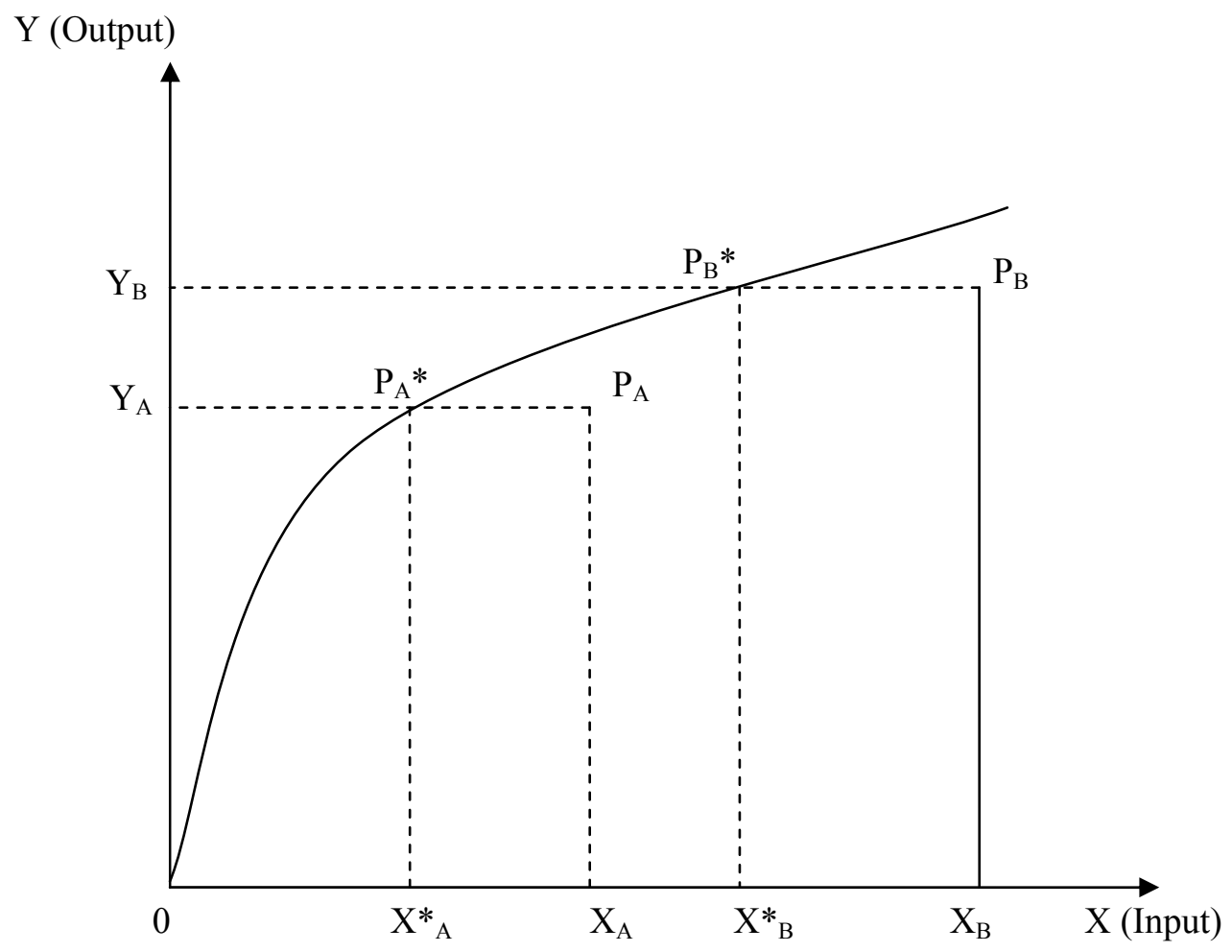

Figure 2 Measuring Input-oriented Technical Efficiency 


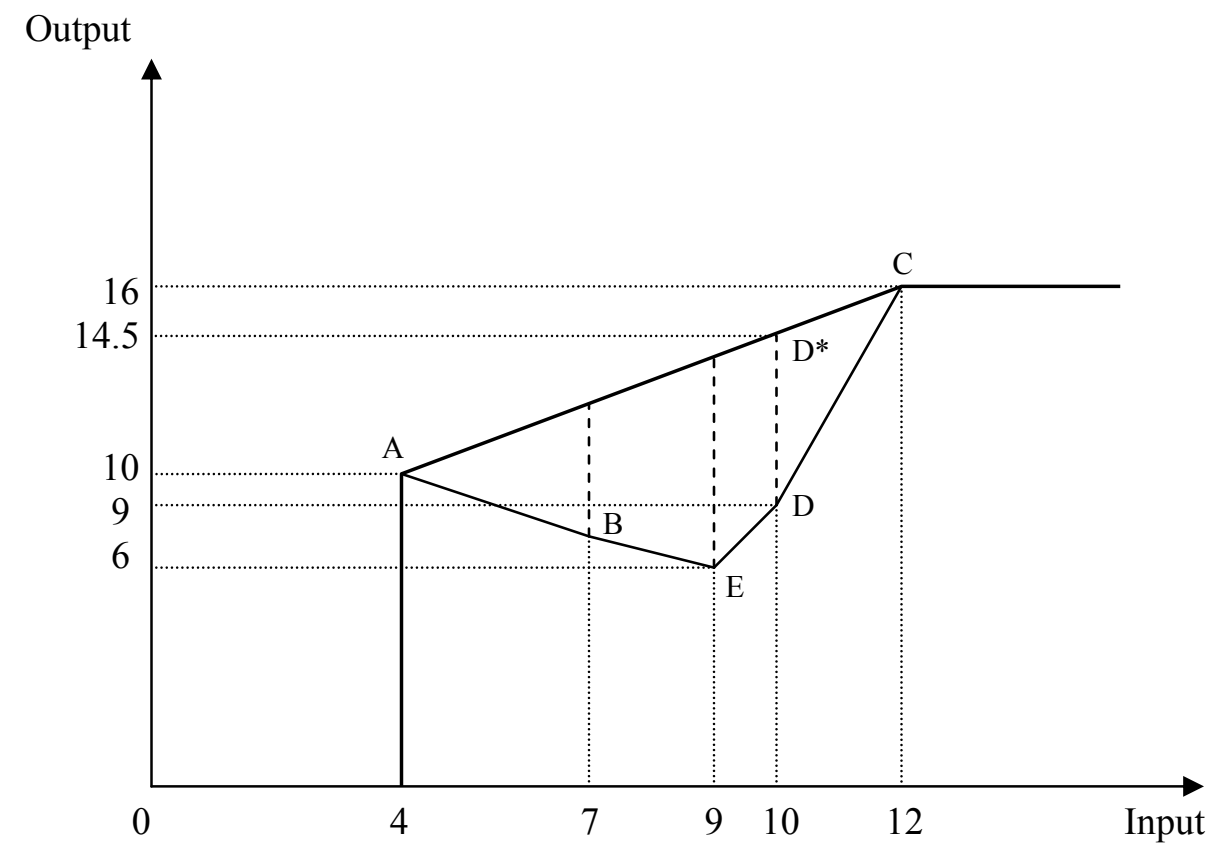

Figure 3. Geometry of DEA 


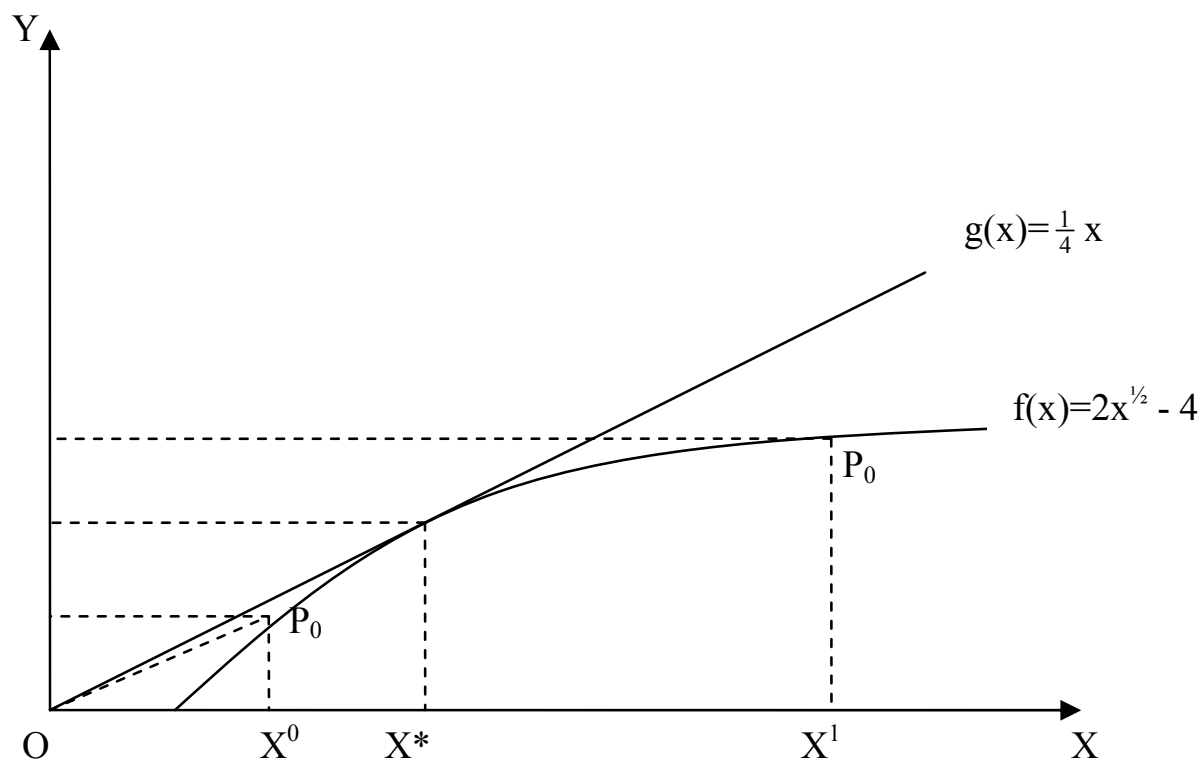

Figure 4 Measuring Scale Efficiency 


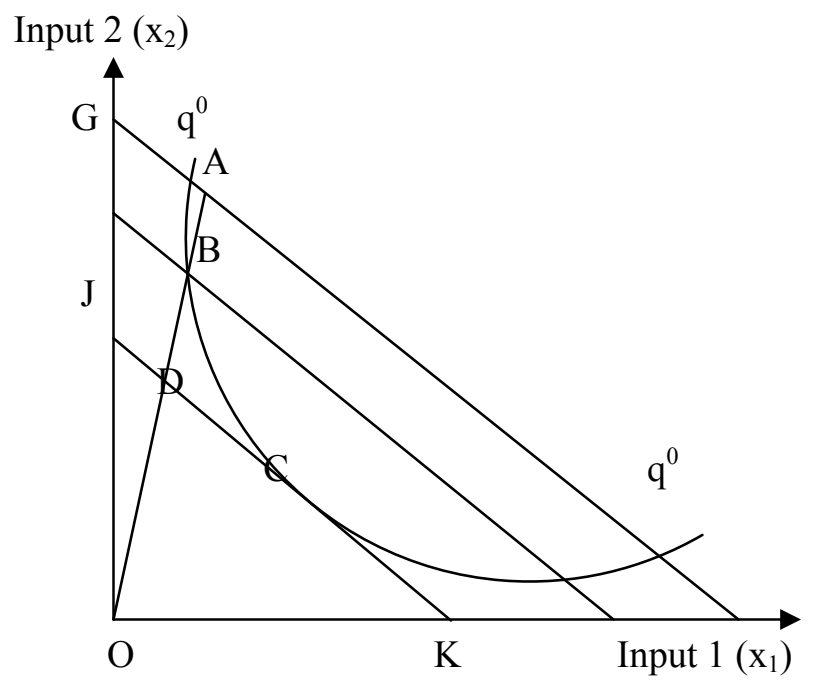

Figure 5 Cost Efficiency and its Decomposition 


\section{References:}

Aigner, D.J., C.A.K. Lovell, and P. Schmidt (1977) “ Formulation and Estimation of Stochastic Frontier Production Function Models"; Journal of Econometrics, 6:1, 21-37.

Banker, R.D., A. Charnes, and W.W. Cooper (1984), "Some Models for Estimating Technical and Scale Inefficiencies in Data Envelopment Analysis," Management Science, 30:9 (September), 1078-92.

Charnes, A., W.W. Cooper, and E. Rhodes (1978) "Measuring the Efficiency of Decision Making Units," European Journal of Operational Research 2:6 (November), 429-44.

Cooper, W.W., L. Seiford and K. Tone.(2007) Data Envelopment Analysis: A Comprehensive Text with Uses, Example Applications, References and DEA-Solver Software. (Norwell, Mass: Kluwer Academic Publishers.)

Färe, R., S. Grosskopf, and C.A.K. Lovell (1994) Production Frontiers (Cambridge: Cambridge University Press).

Kumbhakar, S. and C.A.K. Lovell (2000) Stochastic Frontier Analysis (New York: Cambridge University Press).

Powell, S.G. and K.R. Baker (2007) Management Science: The Art of Modeling with Spreadsheets (New York: John Wiley).

Ray, S.C. (2004) Data Envelopment Analysis: Theory and Techniques for Economics and Operations Research (New York: Cambridge University Press). 